\title{
Stability of delayed HIV dynamics models with two latent reservoirs and immune impairment
}

\author{
A.M. Elaiw' ${ }^{1 *}$ (D, A.A. Raezah ${ }^{1,2}$ and S.A. Azoz ${ }^{3}$
}

"Correspondence:

a_m_elaiw@yahoo.com

1 Department of Mathematics,

Faculty of Science, King Abdulaziz

University, Jeddah, Saudi Arabia

Full list of author information is

available at the end of the article

\begin{abstract}
The purpose of this paper is to investigate the qualitative behavior of HIV dynamics models with immune impairment. The HIV particles infect both $\mathrm{CD}^{+} \mathrm{T}$ cells and macrophages. Both latently and actively infected cells are incorporated into the models. The models consider multiple discrete or distributed delays to characterize the time between an HIV contact of an uninfected target cell and the creation of mature HIV. The existence and global stability of the steady states are determined by the basic reproduction number. The global stability analysis of the steady states is performed using Lyapunov method. We solve the system of delay differential equations numerically to support the theoretical results.
\end{abstract}

Keywords: HIV infection; Immune impairment; Global stability; Time delay

\section{Introduction}

In order to understand the Human Immunodeficiency Virus (HIV) dynamics within-host and the drug therapy strategies, many mathematical models have been constructed and developed (see [1-40]). Some of these models assume that HIV infects one category of target cells, that is, $C D 4^{+} \mathrm{T}$ cells $[1,29,40]$. Among these models, the basic model which has been developed by Nowak and Bangham [1] is considered the most popular. The model is a three-dimensional system of ODEs which characterize the interaction between HIV $(v)$, uninfected $\mathrm{CD} 4^{+} \mathrm{T}$ cells $(x)$, and infected $\mathrm{CD} 4^{+} \mathrm{T}$ cells $(u)$. Other HIV dynamics models assume that HIV has categories of target cells, $\mathrm{CD}^{+} \mathrm{T}$ cells and macrophages [30-40]. The basic model describing the HIV interaction with two categories of target cells is presented in [30] and [32] as:

$$
\begin{aligned}
& \dot{x}_{1}(t)=\lambda_{1}-d_{1} x_{1}(t)-\beta_{1} x_{1}(t) v(t), \\
& \dot{u}_{1}(t)=\beta_{1} x_{1}(t) v(t)-\mu_{1} u_{1}(t), \\
& \dot{x}_{2}(t)=\lambda_{2}-d_{2} x_{2}(t)-\beta_{2} x_{2}(t) v(t), \\
& \dot{u}_{2}(t)=\beta_{2} x_{2}(t) v(t)-\mu_{2} u_{2}(t), \\
& \dot{v}(t)=k_{1} u_{1}(t)+k_{2} u_{2}(t)-r v(t) .
\end{aligned}
$$

(c) The Author(s) 2018. This article is distributed under the terms of the Creative Commons Attribution 4.0 International License (http://creativecommons.org/licenses/by/4.0/), which permits unrestricted use, distribution, and reproduction in any medium, provided you give appropriate credit to the original author(s) and the source, provide a link to the Creative Commons license, and indicate if changes were made. 
The variables describe the concentrations of $x_{i}$, the uninfected and infected cells and free HIV, respectively; $i=1$ and $i=2$ represent the $C D 4^{+} \mathrm{T}$ cells and macrophages, respectively; $\lambda_{i}$ represents the generation rate of the uninfected cells; $d_{i}$ are the death rate constants; and $\beta_{i}$ are the infection rate constants. Equations (2) and (4) represent the population dynamics of the activated infected cells and show that they die with the same constant rate $\mu_{i}$. The HIV particles are generated from infected CD $4^{+} \mathrm{T}$ cells and infected macrophages at rates $k_{1} u_{1}$ and $k_{2} u_{2}$, respectively. The HIV particles are cleared at rate $r v$.

A lot of considerations have been added that aim to get the best representation of the HIV infection. Most notable are latent HIV reservoirs which serve as a major barrier in curing HIV infection. Despite the fact that the antiretroviral therapy significantly limits the level of HIV in the blood, there is still a low viral load due to ongoing reactivation of latent infected cells reservoirs. Variant models have been developed to study the dynamics of HIV in the presence of latent reservoirs (see, e.g., [23-34]).

Cytotoxic T Lymphocyte (CTL) cells play a prominent role in achieving the best representation of the HIV dynamics. CTL cells attack the HIV infected cells and hence try to eliminate or control the infection. The first mathematical model describing the interaction between CTL immune response and viral infection had been presented by Nowak and Bangham [1], since then many mathematical models have been presented to study the effect of CTL immune response on virus dynamics (see, e.g., [41-44]). However, it has been reported in [45] that during the infection, HIV causes an impairment in the CTL job. Virus dynamics models with CTL immune impairment have been studied in many papers (see, e.g., [45-52]). All the HIV dynamics models with CTL immune impairment presented in the literature consider one category of target cells, $\mathrm{CD} 4^{+} \mathrm{T}$ cells.

In the present paper we investigate the global stability of an HIV dynamics model with CTL immune impairment and two categories of target cells, CD4 $4^{+} \mathrm{T}$ cells and macrophages. The importance of considering such a model is due to the observation of Perleson el al. that after the rapid first phase of decay during the initial 1-2 weeks of an antiretroviral treatment, plasma virus levels declined at a considerably slower rate [31]. This second phase of viral decay was attributed to the turnover of a longer-lived virus reservoir of infected cell population. Therefore, the two target cells model is more accurate than the one target cell model, and then more accurate drug efficacy is determined when using the model with two classes of target cells. Moreover, a better understanding of the mechanisms behind this immune impairment in HIV infection may help to devise better therapeutic regimens and to identify patients more responsive to certain drugs [53]. We consider both latently and actively infected cells. We incorporate multiple discrete or distributed time delays to describe the time between the HIV contacts of target cells and the emission of new mature HIV particles. We study global stability of the two steady states of the model using Lyapunov method. The theoretical results are supported by numerical simulations.

\section{HIV model with discrete delays}

We study the following HIV model:

$$
\begin{aligned}
& \dot{x}_{1}(t)=\lambda_{1}-d_{1} x_{1}(t)-\beta_{1} x_{1}(t) v(t), \\
& \dot{y}_{1}(t)=\rho_{1} \beta_{1} e^{-\alpha_{1} \tau_{1}} x_{1}\left(t-\tau_{1}\right) v\left(t-\tau_{1}\right)-a y_{1}(t)-\delta y_{1}(t),
\end{aligned}
$$




$$
\begin{aligned}
& \dot{u}_{1}(t)=\left(1-\rho_{1}\right) \beta_{1} e^{-\alpha_{2} \tau_{2}} x_{1}\left(t-\tau_{2}\right) v\left(t-\tau_{2}\right)+\delta y_{1}(t)-p u_{1}(t) z(t)-\mu u_{1}(t), \\
& \dot{x}_{2}(t)=\lambda_{2}-d_{2} x_{2}(t)-\beta_{2} x_{2}(t) v(t), \\
& \dot{y}_{2}(t)=\rho_{2} \beta_{2} e^{-\alpha_{3} \tau_{3}} x_{2}\left(t-\tau_{3}\right) v\left(t-\tau_{3}\right)-a y_{2}(t)-\delta y_{2}(t), \\
& \dot{u}_{2}(t)=\left(1-\rho_{2}\right) \beta_{2} e^{-\alpha_{4} \tau_{4}} x_{2}\left(t-\tau_{4}\right) v\left(t-\tau_{4}\right)+\delta y_{2}(t)-p u_{2}(t) z(t)-\mu u_{2}(t), \\
& \dot{v}(t)=k e^{-\alpha_{5} \tau_{5}}\left(u_{1}\left(t-\tau_{5}\right)+u_{2}\left(t-\tau_{5}\right)\right)-r v(t), \\
& \dot{z}(t)=c\left(u_{1}(t)+u_{2}(t)\right)-m\left(u_{1}(t)+u_{2}(t)\right) z(t)-b z(t) .
\end{aligned}
$$

Here $u_{i}, y_{i}$ and $z$ are the concentrations of the latent infected, actively infected and CTL cells, respectively. The fractions $\rho_{i}$ and $1-\rho_{i}$ with $0<\rho_{i}<1$ where $i=1,2$ are the probabilities that upon infection, uninfected cell will become either latently or actively infected. Equations (7) and (10) describe the population dynamics of the latent infected CD4 ${ }^{+} \mathrm{T}$ and macrophages cells and shows that they die with the same constant rate $a$ and become active with constant rate $\delta y_{i}$. The actively infected cells are removed by the CTL at rate $p u_{i} z$. The CTLs are proliferated at the rate of $c\left(u_{1}+u_{2}\right)$ and decay at rate of $b z$. The term $m\left(u_{1}+u_{2}\right) z$ represents the immune impairment rate. Here $\tau_{1}$ and $\tau_{3}$ are the times between HIV entry of $\mathrm{CD} 4^{+} \mathrm{T}$ cells and macrophages, respectively, to become latent infected; $\tau_{2}$ and $\tau_{4}$ are the times between HIV entry of $\mathrm{CD} 4^{+} \mathrm{T}$ cells and macrophages, respectively, to produce immature HIV. The immature HIVs need time $\tau_{5}$ to become mature. The factors $e^{-\alpha_{j} \tau_{j}}, j=1, \ldots, 5$, represent the probability of surviving to the age of $\tau_{i}$, where $\alpha_{j}$ are positive constants.

To ensure the uniqueness of the solution of system (6)-(13), we take the following initial conditions [54]:

$$
\begin{aligned}
& x_{1}(\eta)=\omega_{1}(\eta), \quad y_{1}(\eta)=\omega_{2}(\eta), \quad u_{1}(\eta)=\omega_{3}(\eta), \\
& x_{2}(\eta)=\omega_{4}(\eta), \quad y_{2}(\eta)=\omega_{5}(\eta), \quad u_{2}(\eta)=\omega_{6}(\eta), \\
& v(\eta)=\omega_{7}(\eta), \quad z(\eta)=\omega_{8}(\eta), \quad \omega_{i}(\eta) \geq 0, \quad \eta \in[-\kappa, 0], i=1, \ldots, 8,
\end{aligned}
$$

where $\kappa=\max \left\{\tau_{1}, \tau_{2}, \tau_{3}, \tau_{4}, \tau_{5}\right\},\left(\omega_{1}(\eta), \ldots, \omega_{8}(\eta)\right) \in C\left([-\kappa, 0], \mathbb{R}_{\geq 0}^{8}\right), C$ is the Banach space of continuous functions mapping the interval $[-\kappa, 0]$ into $\mathbb{R}_{\geq 0}$.

\subsection{Nonnegativity and boundedness}

Proposition 1 The solutions of system (6)-(13) are nonnegative and ultimately bounded.

Proof From Eqs. (6)-(13) of the system, we have

$$
\begin{aligned}
& x_{i}(t) \geq \omega_{3 i-2}(0) e^{-\int_{0}^{t}\left(d_{i}+\beta_{i} v(s)\right) d s}, \quad i=1,2, \\
& y_{i}(t) \geq \omega_{3 i-1}(0) e^{-(a+\delta) t}, \quad i=1,2, \\
& u_{i}(t) \geq \omega_{3 i}(0) e^{-\int_{0}^{t}(\mu+p z(s)) d s}, \quad i=1,2, \\
& v(t) \geq \omega_{7}(0) e^{-r t}, \\
& z(t) \geq \omega_{8}(0) e^{-\int_{0}^{t}\left(m\left(u_{1}(s)+u_{2}(s)\right)+b\right) d s},
\end{aligned}
$$

therefore $x_{i}(t), y_{i}(t), u_{i}(t), v(t), z(t) \geq 0$ for all $t \geq 0$ and $i=1,2$. 
From Eqs. (6) and (9), we have $\sup _{t \rightarrow \infty} x_{i}(t) \leq \frac{\lambda_{i}}{d_{i}}$. Let us define

$$
\begin{aligned}
L_{1}(t)= & \rho_{1} e^{-\alpha_{1} \tau_{1}} x_{1}\left(t-\tau_{1}\right)+\left(1-\rho_{1}\right) e^{-\alpha_{2} \tau_{2}} x_{1}\left(t-\tau_{2}\right)+\rho_{2} e^{-\alpha_{3} \tau_{3}} x_{2}\left(t-\tau_{3}\right) \\
& +\left(1-\rho_{2}\right) e^{-\alpha_{4} \tau_{4}} x_{2}\left(t-\tau_{4}\right)+y_{1}(t)+u_{1}(t)+y_{2}(t)+u_{2}(t)+\frac{\mu}{2 c} z(t) .
\end{aligned}
$$

Then

$$
\begin{aligned}
& \dot{L}_{1}(t)=\rho_{1} e^{-\alpha_{1} \tau_{1}}\left[\lambda_{1}-d_{1} x_{1}\left(t-\tau_{1}\right)-\beta_{1} x_{1}\left(t-\tau_{1}\right) v\left(t-\tau_{1}\right)\right] \\
& +\left(1-\rho_{1}\right) e^{-\alpha_{2} \tau_{2}}\left[\lambda_{1}-d_{1} x_{1}\left(t-\tau_{2}\right)-\beta_{1} x_{1}\left(t-\tau_{2}\right) v\left(t-\tau_{2}\right)\right] \\
& +\rho_{2} e^{-\alpha_{3} \tau_{3}}\left[\lambda_{2}-d_{2} x_{2}\left(t-\tau_{3}\right)-\beta_{2} x_{2}\left(t-\tau_{3}\right) v\left(t-\tau_{3}\right)\right] \\
& +\left(1-\rho_{2}\right) e^{-\alpha_{4} \tau_{4}}\left[\lambda_{2}-d_{2} x_{2}\left(t-\tau_{4}\right)-\beta_{2} x_{2}\left(t-\tau_{4}\right) v\left(t-\tau_{4}\right)\right] \\
& +\rho_{1} \beta_{1} e^{-\alpha_{1} \tau_{1}} x_{1}\left(t-\tau_{1}\right) v\left(t-\tau_{1}\right)-a y_{1}(t)-\delta y_{1}(t) \\
& +\left(1-\rho_{1}\right) \beta_{1} e^{-\alpha_{2} \tau_{2}} x_{1}\left(t-\tau_{2}\right) v\left(t-\tau_{2}\right)+\delta y_{1}(t)-p u_{1}(t) z(t)-\mu u_{1}(t) \\
& +\rho_{2} \beta_{2} e^{-\alpha_{3} \tau_{3}} x_{2}\left(t-\tau_{3}\right) v\left(t-\tau_{3}\right)-a y_{2}(t)-\delta y_{2}(t) \\
& +\left(1-\rho_{2}\right) \beta_{2} e^{-\alpha_{4} \tau_{4}} x_{2}\left(t-\tau_{4}\right) v\left(t-\tau_{4}\right)+\delta y_{2}(t)-p u_{2}(t) z(t)-\mu u_{2}(t) \\
& +\frac{\mu}{2}\left(u_{1}(t)+u_{2}(t)\right)-\frac{\mu m}{2 c}\left(u_{1}(t)+u_{2}(t)\right) z(t)-\frac{\mu b}{2 c} z(t) \\
& =\rho_{1} e^{-\alpha_{1} \tau_{1}} \lambda_{1}+\left(1-\rho_{1}\right) e^{-\alpha_{2} \tau_{2}} \lambda_{1}+\rho_{2} e^{-\alpha_{3} \tau_{3}} \lambda_{2}+\left(1-\rho_{2}\right) e^{-\alpha_{4} \tau_{4}} \lambda_{2} \\
& -\left(p+\frac{\mu m}{2 c}\right)\left(u_{1}(t)+u_{2}(t)\right) z(t)-\rho_{1} e^{-\alpha_{1} \tau_{1}} d_{1} x_{1}\left(t-\tau_{1}\right) \\
& -\left(1-\rho_{1}\right) e^{-\alpha_{2} \tau_{2}} d_{1} x_{1}\left(t-\tau_{2}\right) \\
& -\rho_{2} e^{-\alpha_{3} \tau_{3}} d_{2} x_{2}\left(t-\tau_{3}\right)-\left(1-\rho_{2}\right) e^{-\alpha_{4} \tau_{4}} d_{2} x_{2}\left(t-\tau_{4}\right)-a y_{1}(t)-a y_{2}(t) \\
& -\frac{\mu}{2} u_{1}(t)-\frac{\mu}{2} u_{2}(t)-\frac{\mu b}{2 c} z(t) \\
& \leq \lambda_{1}+\lambda_{2}-\delta_{1} L_{1}(t)
\end{aligned}
$$

$\delta_{1}=\min \left\{d_{1}, d_{2}, a, \frac{\mu}{2}, b\right\}$. Hence $\limsup _{t \rightarrow \infty} L_{1}(t) \leq M_{1}, \quad \limsup _{t \rightarrow \infty} y_{i}(t) \leq M_{1}$, $\limsup \sup _{t \rightarrow \infty} u_{i}(t) \leq M_{1}$ and $\limsup _{t \rightarrow \infty} z(t) \leq M_{2}$, for all $t \geq 0$ where $i=1,2$, where, $M_{1}=\frac{\lambda_{1}+\lambda_{2}}{\delta_{1}}$ and $M_{2}=\frac{2 c}{\mu}$. From Eq. (12) we have

$$
\begin{aligned}
\dot{v}(t) & =k e^{-\alpha_{5} \tau_{5}}\left(u_{1}\left(t-\tau_{5}\right)+u_{2}\left(t-\tau_{5}\right)\right)-r v(t) \\
& \leq 2 k M_{1}-r v(t)
\end{aligned}
$$

which yields $\lim \sup _{t \rightarrow \infty} v(t) \leq M_{3}$ where $M_{3}=\frac{2 k M_{1}}{r}$.

\subsection{Steady states}

The basic reproduction number of system (6)-(13) is given as:

$$
R_{0}=\frac{\delta k}{\mu r(a+\delta)}\left(\gamma_{1} \beta_{1} x_{1}^{0}+\gamma_{2} \beta_{2} x_{2}^{0}\right)
$$


where

$$
\begin{aligned}
& \gamma_{1}=\rho_{1} e^{-\alpha_{1} \tau_{1}-\alpha_{5} \tau_{5}}+\frac{(a+\delta)}{\delta}\left(1-\rho_{1}\right) e^{-\alpha_{2} \tau_{2}-\alpha_{5} \tau_{5}}, \\
& \gamma_{2}=\rho_{2} e^{-\alpha_{3} \tau_{3}-\alpha_{5} \tau_{5}}+\frac{(a+\delta)}{\delta}\left(1-\rho_{2}\right) e^{-\alpha_{4} \tau_{4}-\alpha_{5} \tau_{5}}, \\
& x_{1}^{0}=\frac{\lambda_{1}}{d_{1}}, \quad x_{2}^{0}=\frac{\lambda_{2}}{d_{2}} .
\end{aligned}
$$

Lemma 1 For system (6)-(13) (i) if $R_{0} \leq 1$, then there exists an infection-free steady state $E_{0}$, and (ii) if $R_{0}>1$, then there exist two steady states, $E_{0}$ and a chronic steady state $E^{*}$.

Proof The steady states of model (6)-(13) satisfy

$$
\begin{aligned}
& \lambda_{1}-d_{1} x_{1}-\beta_{1} x_{1} v=0, \\
& \rho_{1} \beta_{1} e^{-\alpha_{1} \tau_{1}} x_{1} v-a y_{1}-\delta y_{1}=0, \\
& \left(1-\rho_{1}\right) \beta_{1} e^{-\alpha_{2} \tau_{2}} x_{1} v+\delta y_{1}-p u_{1} z-\mu u_{1}=0, \\
& \lambda_{2}-d_{2} x_{2}-\beta_{2} x_{2} v=0, \\
& \rho_{2} \beta_{2} e^{-\alpha_{3} \tau_{3}} x_{2} v-a y_{2}-\delta y_{2}=0, \\
& \left(1-\rho_{2}\right) \beta_{2} e^{-\alpha_{4} \tau_{4}} x_{2} v+\delta y_{2}-p u_{2} z-\mu u_{2}=0, \\
& k e^{-\alpha_{5} \tau_{5}}\left(u_{1}+u_{2}\right)-r v=0, \\
& c\left(u_{1}+u_{2}\right)-m\left(u_{1}+u_{2}\right) z-b z=0 .
\end{aligned}
$$

One solution of Eqs. (15)-(22) yields an infection-free steady state $E_{0}=\left(x_{1}^{0}, 0,0, x_{2}^{0}, 0,0\right.$, $0,0)$. Moreover, we have

$$
A_{1} v^{3}+B_{1} v^{2}+C_{1} v+D_{1}=0
$$

where

$$
\begin{aligned}
A_{1}= & \beta_{1} \beta_{2}(\mu m+p c) r^{2}, \\
B_{1}= & \beta_{1} \beta_{2} \mu r b k e^{-\alpha_{5} \tau_{5}}+\left(\beta_{1} d_{2}+\beta_{2} d_{1}\right)(\mu m+p c) r^{2}-\frac{\beta_{1} \beta_{2} m r \delta k}{a+\delta}\left(\lambda_{1} \gamma_{1}+\lambda_{2} \gamma_{2}\right), \\
C_{1}= & \mu r b k e^{-\alpha_{5} \tau_{5}}\left(\beta_{1} d_{2}+\beta_{2} d_{1}\right)+\mu m r^{2} d_{1} d_{2}\left(1-R_{0}\right)+p c r^{2} d_{1} d_{2} \\
& -\frac{\beta_{1} \beta_{2} b k^{2} \delta e^{-\alpha_{5} \tau_{5}}}{a+\delta}\left(\lambda_{1} \gamma_{1}+\lambda_{2} \gamma_{2}\right), \\
& =\mu r b k d_{1} d_{2} e^{-\alpha_{5} \tau_{5}}\left(1-R_{0}\right) .
\end{aligned}
$$

Let

$$
\Psi_{1}(v)=A_{1} v^{3}+B_{1} v^{2}+C_{1} v+D_{1}=0 .
$$

If $R_{0}>1$, then $\Psi_{1}(0)=D_{1}<0$; moreover, $\lim _{v \rightarrow \infty} \Psi_{1}(v)=\infty$, which implies that there exists $v^{*}>0$ such that $\Psi_{1}\left(v^{*}\right)=0$. Thus a chronic steady state $E^{*}=\left(x_{1}^{*}, y_{1}^{*}, u_{1}^{*}, x_{2}^{*}, y_{2}^{*}, u_{2}^{*}, v^{*}, z^{*}\right)$ 
exists when $R_{0}>1$, where

$$
\begin{aligned}
& x_{i}^{*}=\frac{\lambda_{i}}{d_{i}+\beta_{i} v^{*}}, \quad i=1,2, \\
& y_{1}^{*}=\frac{\rho_{1} \beta_{1} e^{-\alpha_{1} \tau_{1}} x_{1}^{*} v^{*}}{a+\delta}, \quad y_{2}^{*}=\frac{\rho_{2} \beta_{2} e^{-\alpha_{3} \tau_{3}} x_{2}^{*} v^{*}}{a+\delta}, \\
& u_{i}^{*}=\frac{\gamma_{i} \beta_{i} x_{i}^{*} v^{*} \delta}{e^{-\alpha_{5} \tau_{5}}\left(\mu+p z^{*}\right)(a+\delta)}, \quad \sum_{i=1}^{2} u_{i}^{*}=\frac{r v^{*}}{e^{-\alpha_{5} \tau_{5} k}}, \\
& z^{*}=\frac{c r v^{*}}{b k e^{-\alpha_{5} \tau_{5}+m r v^{*}} .}
\end{aligned}
$$

\subsection{Global stability}

We use Lyapunov method to investigate the global stability of the steady states. Let $f(\eta)=$ $\eta-1-\ln (\eta)$ and $\left(x_{1}, y_{1}, u_{1}, x_{2}, y_{2}, u_{2}, v, z\right)=\left(x_{1}(t), y_{1}(t), u_{1}(t), x_{2}(t), y_{2}(t), u_{2}(t), v(t), z(t)\right)$.

Theorem 1 If $R_{0} \leq 1$, then $E_{0}$ for system (6)-(13) is globally asymptotically stable.

Proof We consider $W_{1}\left(x_{1}, y_{1}, u_{1}, x_{2}, y_{2}, u_{2}, v, z\right)$ as

$$
\begin{aligned}
W_{1}= & \sum_{i=1}^{2}\left[\gamma_{i} x_{i}^{0} f\left(\frac{x_{i}}{x_{i}^{0}}\right)+e^{-\alpha_{5} \tau_{5}} y_{i}+\frac{a+\delta}{\delta} e^{-\alpha_{5} \tau_{5}} u_{i}\right]+\frac{\mu(a+\delta)}{\delta k} v+\frac{p(a+\delta)}{2 \delta c} e^{-\alpha_{5} \tau_{5}} z^{2} \\
& +\rho_{1} e^{-\alpha_{1} \tau_{1}-\alpha_{5} \tau_{5}} \int_{0}^{\tau_{1}} \beta_{1} x_{1}(t-\vartheta) v(t-\vartheta) d \vartheta \\
& +\frac{(a+\delta)}{\delta}\left(1-\rho_{1}\right) e^{-\alpha_{2} \tau_{2}-\alpha_{5} \tau_{5}} \int_{0}^{\tau_{2}} \beta_{1} x_{1}(t-\vartheta) v(t-\vartheta) d \vartheta \\
& +\rho_{2} e^{-\alpha_{3} \tau_{3}-\alpha_{5} \tau_{5}} \int_{0}^{\tau_{3}} \beta_{2} x_{2}(t-\vartheta) v(t-\vartheta) d \vartheta \\
& +\frac{(a+\delta)}{\delta}\left(1-\rho_{2}\right) e^{-\alpha_{4} \tau_{4}-\alpha_{5} \tau_{5}} \int_{0}^{\tau_{4}} \beta_{2} x_{2}(t-\vartheta) v(t-\vartheta) d \vartheta \\
& +\frac{\mu(a+\delta)}{\delta} e^{-\alpha_{5} \tau_{5}} \int_{0}^{\tau_{5}}\left[u_{1}(t-\vartheta)+u_{2}(t-\vartheta)\right] d \vartheta .
\end{aligned}
$$

It is seen that $W_{1}>0$ for all $x_{1}, y_{1}, u_{1}, x_{2}, y_{2}, u_{2}, v, z>0$, and $W_{1}\left(x_{1}^{0}, 0,0, x_{2}^{0}, 0,0,0,0\right)=0$. Calculating $\dot{W}_{1}$ along system (6)-(13), we obtain

$$
\begin{aligned}
\dot{W}_{1}= & \sum_{i=1}^{2}\left[\gamma_{i}\left(1-\frac{x_{i}^{0}}{x_{i}}\right) \dot{x}_{i}+e^{-\alpha_{5} \tau_{5}} \dot{y}_{i}+\frac{a+\delta}{\delta} e^{-\alpha_{5} \tau_{5}} \dot{u}_{i}\right] \\
& +\frac{\mu(a+\delta)}{\delta k} \dot{v}+\frac{p(a+\delta)}{\delta c} e^{-\alpha_{5} \tau_{5}} z \dot{z} \\
& +\rho_{1} e^{-\alpha_{1} \tau_{1}-\alpha_{5} \tau_{5}} \beta_{1}\left[x_{1} v-x_{1}\left(t-\tau_{1}\right) v\left(t-\tau_{1}\right)\right] \\
& +\frac{(a+\delta)\left(1-\rho_{1}\right)}{\delta} e^{-\alpha_{2} \tau_{2}-\alpha_{5} \tau_{5}} \beta_{1}\left[x_{1} v-x_{1}\left(t-\tau_{2}\right) v\left(t-\tau_{2}\right)\right] \\
& +\rho_{2} e^{-\alpha_{3} \tau_{3}-\alpha_{5} \tau_{5}} \beta_{2}\left[x_{2} v-x_{2}\left(t-\tau_{3}\right) v\left(t-\tau_{3}\right)\right] \\
& +\frac{(a+\delta)\left(1-\rho_{2}\right)}{\delta} e^{-\alpha_{4} \tau_{4}-\alpha_{5} \tau_{5}} \beta_{2}\left[x_{2} v-x_{2}\left(t-\tau_{4}\right) v\left(t-\tau_{4}\right)\right]
\end{aligned}
$$




$$
+\frac{\mu(a+\delta)}{\delta} e^{-\alpha_{5} \tau_{5}}\left[u_{1}+u_{2}-u_{1}\left(t-\tau_{5}\right)-u_{2}\left(t-\tau_{5}\right)\right]
$$

Equation (23) can be simplified as

$$
\begin{aligned}
\dot{W}_{1}= & \sum_{i=1}^{2}\left[\gamma_{i}\left(1-\frac{x_{i}^{0}}{x_{i}}\right)\left(\lambda_{i}-d_{i} x_{i}-\beta_{i} x_{i} v\right)\right]-\frac{\mu(a+\delta)}{\delta k} r v \\
& +\frac{p(a+\delta)}{\delta c} e^{-\alpha_{5} \tau_{5}} z\left[-m\left(u_{1}+u_{2}\right) z-b z\right]+\gamma_{1} \beta_{1} x_{1} v+\gamma_{2} \beta_{2} x_{2} v .
\end{aligned}
$$

Since $\lambda_{i}=d_{i} x_{i}^{0}$,

$$
\begin{aligned}
\dot{W}_{1}= & \sum_{i=1}^{2}\left[\gamma_{i}\left(1-\frac{x_{i}^{0}}{x_{i}}\right)\left(d_{i} x_{i}^{0}-d_{i} x_{i}\right)-\gamma_{i} \beta_{i} x_{i} v\left(1-\frac{x_{i}^{0}}{x_{i}}\right)+\gamma_{i} \beta_{i} x_{i} v\right]-\frac{\mu(a+\delta)}{\delta k} r v \\
& -\frac{p m(a+\delta)}{\delta c} e^{-\alpha_{5} \tau_{5}}\left(u_{1}+u_{2}\right) z^{2}-\frac{p b(a+\delta)}{\delta c} e^{-\alpha_{5} \tau_{5}} z^{2} \\
= & \sum_{i=1}^{2} \frac{-d_{i} \gamma_{i}\left(x_{i}-x_{i}^{0}\right)^{2}}{x_{i}}+\frac{\mu r(a+\delta)}{\delta k}\left(R_{0}-1\right) v \\
& -\frac{p m(a+\delta) e^{-\alpha_{5} \tau_{5}}}{\delta c} \sum_{i=1}^{2} u_{i} z^{2}-\frac{p b(a+\delta) e^{-\alpha_{5} \tau_{5}}}{\delta c} z^{2} .
\end{aligned}
$$

It follows that $\dot{W}_{1} \leq 0$ if $R_{0} \leq 1$. Therefore, $\dot{W}_{1}=0$ implies that $x_{i}=x_{i}^{0}, v=0$ and $z=0$. One can easily show that the largest invariant set $\Omega_{0} \subseteq \Omega=\left\{\left(x_{1}, y_{1}, u_{1}, x_{2}, y_{2}, u_{2}, v, z\right) \mid \dot{W}_{1}=0\right\}$ is the singleton $\left\{E_{0}\right\}$. By LaSalle's invariance principle, $E_{0}$ is globally asymptotically stable. $\square$

Theorem 2 If $R_{0}>1$, then $E^{*}$ for system (6)-(13) is globally asymptotically stable.

Proof Consider $W_{2}\left(x_{1}, y_{1}, u_{1}, x_{2}, y_{2}, u_{2}, v, z\right)$ as

$$
\begin{aligned}
& W_{2}=\sum_{i=1}^{2}\left[\gamma_{i} x_{i}^{*} f\left(\frac{x_{i}}{x_{i}^{*}}\right)+e^{-\alpha_{5} \tau_{5}} y_{i}^{*} f\left(\frac{y_{i}}{y_{i}^{*}}\right)+\frac{(a+\delta)}{\delta} e^{-\alpha_{5} \tau_{5}} u_{i}^{*} f\left(\frac{u_{i}}{u_{i}^{*}}\right)\right] \\
& +\frac{(a+\delta)\left(\mu+p z^{*}\right)}{\delta k} v^{*} f\left(\frac{v}{v^{*}}\right) \\
& +\frac{p(a+\delta)}{2 \delta\left(c-m z^{*}\right)} e^{-\alpha_{5} \tau_{5}}\left(z-z^{*}\right)^{2}+\rho_{1} \beta_{1} e^{-\alpha_{1} \tau_{1}-\alpha_{5} \tau_{5}} x_{1}^{*} v^{*} \int_{0}^{\tau_{1}} f\left(\frac{x_{1}(t-\vartheta) v(t-\vartheta)}{x_{1}^{*} v^{*}}\right) d \vartheta \\
& +\frac{(a+\delta)}{\delta}\left(1-\rho_{1}\right) \beta_{1} e^{-\alpha_{2} \tau_{2}-\alpha_{5} \tau_{5}} x_{1}^{*} v^{*} \int_{0}^{\tau_{2}} f\left(\frac{x_{1}(t-\vartheta) v(t-\vartheta)}{x_{1}^{*} v^{*}}\right) d \vartheta \\
& +\rho_{2} \beta_{2} e^{-\alpha_{3} \tau_{3}-\alpha_{5} \tau_{5}} x_{2}^{*} v^{*} \int_{0}^{\tau_{3}} f\left(\frac{x_{2}(t-\vartheta) v(t-\vartheta)}{x_{2}^{*} \nu^{*}}\right) d \vartheta \\
& +\frac{(a+\delta)}{\delta}\left(1-\rho_{2}\right) \beta_{2} e^{-\alpha_{4} \tau_{4}-\alpha_{5} \tau_{5}} x_{2}^{*} v^{*} \int_{0}^{\tau_{4}} f\left(\frac{x_{2}(t-\vartheta) \nu(t-\vartheta)}{x_{2}^{*} v^{*}}\right) d \vartheta \\
& +\frac{(a+\delta)\left(\mu+p z^{*}\right)}{\delta} e^{-\alpha_{5} \tau_{5}} \int_{0}^{\tau_{5}}\left[u_{1}^{*} f\left(\frac{u_{1}(t-\vartheta)}{u_{1}^{*}}\right)+u_{2}^{*} f\left(\frac{u_{2}(t-\vartheta)}{u_{2}^{*}}\right)\right] d \vartheta
\end{aligned}
$$


Elaiw et al. Advances in Difference Equations

(2018) 2018:414

Page 8 of 25

Calculating $\dot{W}_{2}$ along system (6)-(13), we obtain

$$
\begin{aligned}
& \dot{W}_{2}=\sum_{i=1}^{2}\left[\gamma_{i}\left(1-\frac{x_{i}^{*}}{x_{i}}\right) \dot{x}_{i}+e^{-\alpha_{5} \tau_{5}}\left(1-\frac{y_{i}^{*}}{y_{i}}\right) \dot{y}_{i}+\frac{(a+\delta)}{\delta} e^{-\alpha_{5} \tau_{5}}\left(1-\frac{u_{i}^{*}}{u_{i}}\right) \dot{u}_{i}\right] \\
& +\frac{(a+\delta)\left(\mu+p z^{*}\right)}{\delta k}\left(1-\frac{v^{*}}{v}\right) \dot{v} \\
& +\frac{p(a+\delta)}{\delta\left(c-m z^{*}\right)} e^{-\alpha_{5} \tau_{5}}\left(z-z^{*}\right) \dot{z} \\
& +\rho_{1} \beta_{1} e^{-\alpha_{1} \tau_{1}-\alpha_{5} \tau_{5}} x_{1}^{*} v^{*}\left[\frac{x_{1} v}{x_{1}^{*} v^{*}}-\frac{x_{1}\left(t-\tau_{1}\right) v\left(t-\tau_{1}\right)}{x_{1}^{*} v^{*}}+\ln \frac{x_{1}\left(t-\tau_{1}\right) v\left(t-\tau_{1}\right)}{x_{1} v}\right] \\
& +\frac{(a+\delta)}{\delta}\left(1-\rho_{1}\right) \beta_{1} e^{-\alpha_{2} \tau_{2}-\alpha_{5} \tau_{5}} x_{1}^{*} v^{*} \\
& \times\left[\frac{x_{1} v}{x_{1}^{*} v^{*}}-\frac{x_{1}\left(t-\tau_{2}\right) v\left(t-\tau_{2}\right)}{x_{1}^{*} v^{*}}+\ln \frac{x_{1}\left(t-\tau_{2}\right) v\left(t-\tau_{2}\right)}{x_{1} v}\right] \\
& +\rho_{2} \beta_{2} e^{-\alpha_{3} \tau_{3}-\alpha_{5} \tau_{5}} x_{2}^{*} v^{*}\left[\frac{x_{2} v}{x_{2}^{*} v^{*}}-\frac{x_{2}\left(t-\tau_{3}\right) v\left(t-\tau_{3}\right)}{x_{2}^{*} v^{*}}+\ln \frac{x_{2}\left(t-\tau_{3}\right) v\left(t-\tau_{3}\right)}{x_{2} v}\right] \\
& +\frac{(a+\delta)}{\delta}\left(1-\rho_{2}\right) \beta_{2} e^{-\alpha_{4} \tau_{4}-\alpha_{5} \tau_{5}} x_{2}^{*} v^{*} \\
& \times\left[\frac{x_{2} v}{x_{2}^{*} v^{*}}-\frac{x_{2}\left(t-\tau_{4}\right) v\left(t-\tau_{4}\right)}{x_{2}^{*} v^{*}}+\ln \frac{x_{2}\left(t-\tau_{4}\right) v\left(t-\tau_{4}\right)}{x_{2} v}\right] \\
& +\frac{(a+\delta)\left(\mu+p z^{*}\right)}{\delta} e^{-\alpha_{5} \tau_{5}}\left[u_{1}^{*}\left(\frac{u_{1}}{u_{1}^{*}}-\frac{u_{1}\left(t-\tau_{5}\right)}{u_{1}^{*}}+\ln \frac{u_{1}\left(t-\tau_{5}\right)}{u_{1}}\right)\right. \\
& \left.+u_{2}^{*}\left(\frac{u_{2}}{u_{2}^{*}}-\frac{u_{2}\left(t-\tau_{5}\right)}{u_{2}^{*}}+\ln \frac{u_{2}\left(t-\tau_{5}\right)}{u_{2}}\right)\right] \text {. }
\end{aligned}
$$

Using the following chronic steady state conditions:

$$
\begin{aligned}
& \lambda_{i}=d_{i} x_{i}^{*}+\beta_{i} x_{i}^{*} v^{*}, \quad(a+\delta) y_{1}^{*}=\rho_{1} \beta_{1} e^{-\alpha_{1} \tau_{1}} x_{1}^{*} v^{*}, \\
& \lambda_{i}=d_{i} x_{i}^{*}+\beta_{i} x_{i}^{*} v^{*}, \quad(a+\delta) y_{1}^{*}=\rho_{1} \beta_{1} e^{-\alpha_{1} \tau_{1}} x_{1}^{*} v^{*}, \\
& (a+\delta) y_{2}^{*}=\rho_{2} \beta_{2} e^{-\alpha_{3} \tau_{3}} x_{2}^{*} v^{*}, \quad(a+\delta)\left(\mu+p z^{*}\right) e^{-\alpha_{5} \tau_{5}} u_{i}^{*}=\delta \gamma_{i} \beta_{i} x_{i}^{*} v^{*}, \\
& r v^{*}=k e^{-\alpha_{5} \tau_{5}}\left(u_{1}^{*}+u_{2}^{*}\right), \quad c\left(u_{1}^{*}+u_{2}^{*}\right)=m\left(u_{1}^{*}+u_{2}^{*}\right) z^{*}+b z^{*},
\end{aligned}
$$

we can simplify:

$$
\begin{aligned}
& \frac{p}{\left(c-m z^{*}\right)}\left(z-z^{*}\right) \dot{z} \\
& =\frac{p\left(z-z^{*}\right)}{\left(c-m z^{*}\right)}\left(c\left(u_{1}+u_{2}\right)-m\left(u_{1}+u_{2}\right) z-b z\right) \\
& =\frac{p\left(z-z^{*}\right)}{\left(c-m z^{*}\right)}\left(c\left(u_{1}+u_{2}\right)-m\left(u_{1}+u_{2}\right) z-b z-c\left(u_{1}^{*}+u_{2}^{*}\right)+m\left(u_{1}^{*}+u_{2}^{*}\right) z^{*}+b z^{*}\right) \\
& =-\frac{p b}{\left(c-m z^{*}\right)}\left(z-z^{*}\right)^{2}-\frac{p m\left(u_{1}+u_{2}\right)}{\left(c-m z^{*}\right)}\left(z-z^{*}\right)^{2} \\
& \quad+p\left(u_{1}-u_{1}^{*}\right)\left(z-z^{*}\right)+p\left(u_{2}-u_{2}^{*}\right)\left(z-z^{*}\right)
\end{aligned}
$$




$$
=p\left[\sum_{i=1}^{2} u_{i}^{*} z^{*}-\sum_{i=1}^{2} u_{i}^{*} z-\sum_{i=1}^{2} u_{i} z^{*}+\sum_{i=1}^{2} u_{i} z-\frac{\left[b+m\left(u_{1}+u_{2}\right)\right]}{\left(c-m z^{*}\right)}\left(z-z^{*}\right)^{2}\right] .
$$

Due to steady state conditions, we can rewrite

$$
\begin{aligned}
& \dot{W}_{2}=\sum_{i=1}^{2} \frac{-\gamma_{i} d_{i}\left(x_{i}-x_{i}^{*}\right)^{2}}{x_{i}}+\rho_{1} \beta_{1} e^{-\alpha_{1} \tau_{1}-\alpha_{5} \tau_{5}} x_{1}^{*} v^{*}\left[4-\frac{x_{1}^{*}}{x_{1}}-\frac{y_{1}^{*} x_{1}\left(t-\tau_{1}\right) v\left(t-\tau_{1}\right)}{y_{1}(t) x_{1}^{*} v^{*}}\right. \\
& \left.-\frac{y_{1} u_{1}^{*}}{y_{1}^{*} u_{1}}-\frac{u_{1}\left(t-\tau_{5}\right) v^{*}}{u_{1}^{*} v}+\ln \frac{x_{1}\left(t-\tau_{1}\right) v\left(t-\tau_{1}\right)}{x_{1} v}+\ln \frac{u_{1}\left(t-\tau_{5}\right)}{u_{1}}\right] \\
& +\frac{(a+\delta)}{\delta}\left(1-\rho_{1}\right) \beta_{1} e^{-\alpha_{2} \tau_{2}-\alpha_{5} \tau_{5}} x_{1}^{*} v^{*}\left[3-\frac{x_{1}^{*}}{x_{1}}-\frac{u_{1}\left(t-\tau_{5}\right) v^{*}}{u_{1}^{*} v}\right. \\
& \left.-\frac{u_{1}^{*} x_{1}\left(t-\tau_{2}\right) v\left(t-\tau_{2}\right)}{u_{1} x_{1}^{*} v^{*}}+\ln \frac{x_{1}\left(t-\tau_{2}\right) v\left(t-\tau_{2}\right)}{x_{1} v}+\ln \frac{u_{1}\left(t-\tau_{5}\right)}{u_{1}}\right] \\
& +\rho_{2} \beta_{2} e^{-\alpha_{3} \tau_{3}-\alpha_{5} \tau_{5}} x_{2}^{*} v^{*}\left[4-\frac{x_{2}^{*}}{x_{2}}-\frac{y_{2}^{*} x_{2}\left(t-\tau_{3}\right) v\left(t-\tau_{3}\right)}{y_{2} x_{2}^{*} v^{*}}-\frac{y_{2} u_{2}^{*}}{y_{2}^{*} u_{2}}-\frac{u_{2}\left(t-\tau_{5}\right) v^{*}}{u_{2}^{*} v}\right. \\
& \left.+\ln \frac{x_{2}\left(t-\tau_{3}\right) v\left(t-\tau_{3}\right)}{x_{2} v}+\ln \frac{u_{2}\left(t-\tau_{5}\right)}{u_{2}}\right] \\
& +\frac{(a+\delta)}{\delta}\left(1-\rho_{2}\right) \beta_{2} e^{-\alpha_{4} \tau_{4}-\alpha_{5} \tau_{5}} x_{2}^{*} v^{*}\left[3-\frac{x_{2}^{*}}{x_{2}}-\frac{u_{2}\left(t-\tau_{5}\right) v^{*}}{u_{2}^{*} v}-\frac{u_{2}^{*} x_{2}\left(t-\tau_{4}\right) v\left(t-\tau_{4}\right)}{u_{2} x_{2}^{*} v^{*}}\right. \\
& \left.+\ln \frac{u_{2}\left(t-\tau_{5}\right)}{u_{2}}+\ln \frac{x_{2}\left(t-\tau_{4}\right) v\left(t-\tau_{4}\right)}{x_{2} v}\right] \\
& -\frac{(a+\delta)}{\delta} e^{-\alpha_{5} \tau_{5}} \frac{p\left[b+m\left(u_{1}+u_{2}\right)\right]}{\left(c-m z^{*}\right)}\left(z-z^{*}\right)^{2} .
\end{aligned}
$$

Consider the following relations:

$$
\begin{aligned}
& \ln \left(\frac{x_{1}\left(t-\tau_{1}\right) v\left(t-\tau_{1}\right)}{x_{1} v}\right)+\ln \left(\frac{u_{1}\left(t-\tau_{5}\right)}{u_{1}}\right) \\
& \quad=\ln \left(\frac{x_{1}^{*}}{x_{1}}\right)+\ln \left(\frac{y_{1}^{*} x_{1}\left(t-\tau_{1}\right) v\left(t-\tau_{1}\right)}{y_{1} x_{1}^{*} v^{*}}\right)+\ln \left(\frac{y_{1} u_{1}^{*}}{y_{1}^{*} u_{1}}\right)+\ln \left(\frac{u_{1}\left(t-\tau_{5}\right) v^{*}}{u_{1}^{*} v}\right), \\
& \ln \left(\frac{x_{1}\left(t-\tau_{2}\right) v\left(t-\tau_{2}\right)}{x_{1} v}\right)+\ln \left(\frac{u_{1}\left(t-\tau_{5}\right)}{u_{1}}\right) \\
& \quad=\ln \left(\frac{x_{1}^{*}}{x_{1}}\right)+\ln \left(\frac{x_{1}\left(t-\tau_{2}\right) v\left(t-\tau_{2}\right) u_{1}^{*}}{x_{1}^{*} v^{*} u_{1}}\right)+\ln \left(\frac{u_{1}\left(t-\tau_{5}\right) v^{*}}{u_{1}^{*} v}\right) .
\end{aligned}
$$

Using similar relations for the terms containing $\tau_{3}$ and $\tau_{4}$, we finally obtain

$$
\begin{aligned}
\dot{W}_{2}= & \sum_{i=1}^{2}\left[\frac{-\gamma_{i} d_{i}\left(x_{i}-x_{i}^{*}\right)^{2}}{x_{i}}\right. \\
& \left.-\gamma_{i} \beta_{i} x_{i}^{*} v^{*} f\left(\frac{x_{i}^{*}}{x_{i}}\right)\right]-\rho_{1} \beta_{1} e^{-\alpha_{1} \tau_{1}-\alpha_{5} \tau_{5}} x_{1}^{*} v^{*}\left[f\left(\frac{y_{1}^{*} x_{1}\left(t-\tau_{1}\right) v\left(t-\tau_{1}\right)}{y_{1} x_{1}^{*} v^{*}}\right)+f\left(\frac{y_{1} u_{1}^{*}}{y_{1}^{*} u_{1}}\right)\right. \\
& \left.+f\left(\frac{u_{1}\left(t-\tau_{5}\right) v^{*}}{u_{1}^{*} v}\right)\right]-\frac{(a+\delta)}{\delta}\left(1-\rho_{1}\right) \beta_{1} e^{-\alpha_{2} \tau_{2}-\alpha_{5} \tau_{5}} x_{1}^{*} v^{*} \\
& \times\left[f\left(\frac{x_{1}\left(t-\tau_{2}\right) v\left(t-\tau_{2}\right) u_{1}^{*}}{x_{1}^{*} v^{*} u_{1}}\right)+f\left(\frac{u_{1}\left(t-\tau_{5}\right) v^{*}}{u_{1}^{*} v}\right)\right]
\end{aligned}
$$




$$
\begin{aligned}
& -\rho_{2} \beta_{2} e^{-\alpha_{3} \tau_{3}-\alpha_{5} \tau_{5}} x_{2}^{*} v^{*}\left[f\left(\frac{y_{2}^{*} x_{2}\left(t-\tau_{3}\right) v\left(t-\tau_{3}\right)}{y_{2}(t) x_{2}^{*} v^{*}}\right)+f\left(\frac{y_{2} u_{2}^{*}}{y_{2}^{*} u_{2}}\right)+f\left(\frac{u_{2}\left(t-\tau_{5}\right) v^{*}}{u_{2}^{*} v}\right)\right] \\
& -\frac{(a+\delta)}{\delta}\left(1-\rho_{2}\right) \beta_{2} e^{-\alpha_{4} \tau_{4}-\alpha_{5} \tau_{5}} x_{2}^{*} v^{*}\left[f\left(\frac{x_{2}\left(t-\tau_{4}\right) v\left(t-\tau_{4}\right) u_{2}^{*}}{x_{2}^{*} v^{*} u_{2}}\right)+f\left(\frac{u_{2}\left(t-\tau_{5}\right) v^{*}}{u_{2}^{*} v}\right)\right] \\
& -\frac{(a+\delta)}{\delta} \frac{p\left[b+m\left(u_{1}+u_{2}\right)\right]}{\left(c-m z^{*}\right)} e^{-\alpha_{5} \tau_{5}}\left(z-z^{*}\right)^{2} .
\end{aligned}
$$

It follows that $\dot{W}_{2} \leq 0$. Now LaSalle's invariance principle implies that $E^{*}$ is globally asymptotically stable.

\section{HIV model with distributed delays}

We study the HIV infection model with distributed time delays:

$$
\begin{aligned}
\dot{x}_{1}(t)= & \lambda_{1}-d_{1} x_{1}(t)-\beta_{1} x_{1}(t) v(t), \\
\dot{y}_{1}(t)= & \rho_{1} \beta_{1} \int_{0}^{s_{1}} h_{1}(\tau) e^{-m_{1} \tau} x_{1}(t-\tau) v(t-\tau) d \tau-a y_{1}(t)-\delta y_{1}(t), \\
\dot{u}_{1}(t)= & \left(1-\rho_{1}\right) \beta_{1} \int_{0}^{s_{2}} h_{2}(\tau) e^{-m_{2} \tau} x_{1}(t-\tau) v(t-\tau) d \tau \\
& +\delta y_{1}(t)-p u_{1}(t) z(t)-\mu u_{1}(t), \\
\dot{x}_{2}(t)= & \lambda_{2}-d_{2} x_{2}(t)-\beta_{2} x_{2}(t) v(t), \\
\dot{y}_{2}(t)= & \rho_{2} \beta_{2} \int_{0}^{s_{3}} h_{3}(\tau) e^{-m_{3} \tau} x_{2}(t-\tau) v(t-\tau) d \tau-a y_{2}(t)-\delta y_{2}(t), \\
\dot{u}_{2}(t)= & \left(1-\rho_{2}\right) \beta_{2} \int_{0}^{s_{4}} h_{4}(\tau) e^{-m_{4} \tau} x_{2}(t-\tau) v(t-\tau) d \tau \\
& +\delta y_{2}(t)-p u_{2}(t) z(t)-\mu u_{2}(t), \\
\dot{v}(t)= & k \int_{0}^{s_{5}} g(\tau) e^{-n \tau}\left(u_{1}(t-\tau)+u_{2}(t-\tau)\right) d \tau-r v(t), \\
\dot{z}(t)= & c\left(u_{1}(t)+u_{2}(t)\right)-m\left(u_{1}(t)+u_{2}(t)\right) z(t)-b z(t) .
\end{aligned}
$$

The factors $h_{2 i-1}(\tau) e^{-m_{2 i-1} \tau}, i=1,2$ are the probabilities that uninfected cells contacted by HIV at time $t-\tau$ survived $\tau$ time units and become latently infected at time $t$, where $i=$ 1 and $i=2$ represent the CD $4^{+} \mathrm{T}$ cells and macrophages, respectively; $h_{2 i}(\tau) e^{-m_{2 i} \tau}, i=1,2$ are the probabilities that the uninfected cells contacted by HIV at time $t-\tau$ survived $\tau$ time units and become actively infected at time $t ; g(\tau) e^{-n \tau}$ is the probability that an immature pathogen at time $t-\tau$ survived $\tau$ time units to become mature at time $t$. The probability distribution function $h_{i}(\tau), i=1, \ldots, 4$ and $g(\tau)$ are assumed to satisfy $h_{i}(\tau)>0, i=1, \ldots, 4$ and $g(\tau)>0$, as well as

$$
\begin{aligned}
& \int_{0}^{s_{j}} h_{j}(\tau) d \tau=\int_{0}^{s_{5}} g(\tau) d \tau=1, \quad \int_{0}^{s_{j}} h_{j}(\tau) e^{\varepsilon r} d r<\infty, \\
& \int_{0}^{s_{5}} g(\tau) e^{\varepsilon r} d r<\infty, \quad i=1, \ldots, 4, \varepsilon>0 .
\end{aligned}
$$


Let

$$
H_{j}=\int_{0}^{s_{j}} h_{j}(\tau) e^{-m_{j} \tau} d \tau=, \quad G=\int_{0}^{s_{5}} g(\tau) e^{-n \tau} d \tau=G, \quad j=1, \ldots, 4 .
$$

Then $0<H_{j} \leq 1$ and $0<G \leq 1, j=1, \ldots, 4$.

The initial conditions for system (25)-(32) are the same as given by (14) where $\kappa=$ $\max \left\{s_{1}, s_{2}, s_{3}, s_{4}, s_{5}\right\}$.

\subsection{Nonnegativity and boundedness}

Proposition 2 The solutions of system (25)-(32) are nonnegative and ultimately bounded.

Proof The nonnegativity can be shown as in Proposition 1 . To show the boundedness, let us define

$$
\begin{aligned}
U_{1}(t)= & \rho_{1} \int_{0}^{s_{1}} h_{1}(\tau) e^{-m_{1} \tau} x_{1}(t-\tau) d \tau+\left(1-\rho_{1}\right) \int_{0}^{s_{2}} h_{2}(\tau) e^{-m_{2} \tau} x_{1}(t-\tau) d \tau \\
& +\rho_{2} \int_{0}^{s_{3}} h_{3}(\tau) e^{-m_{3} \tau} x_{2}(t-\tau) d \tau+\left(1-\rho_{2}\right) \int_{0}^{s_{4}} h_{4}(\tau) e^{-m_{4} \tau} x_{2}(t-\tau) d \tau \\
& +y_{1}(t)+u_{1}(t)+y_{2}(t)+u_{2}(t)+\frac{\mu}{2 c} z(t) .
\end{aligned}
$$

Then

$$
\begin{aligned}
\dot{U}_{1}(t)= & \rho_{1} H_{1} \lambda_{1}+\left(1-\rho_{1}\right) H_{2} \lambda_{1}+\rho_{2} H_{3} \lambda_{2}+\left(1-\rho_{2}\right) H_{4} \lambda_{2} \\
& -\left(p+\frac{\mu m}{2 c}\right)\left(u_{1}(t)+u_{2}(t)\right) z(t)-\rho_{1} e^{-\alpha_{1} \tau_{1}} d_{1} x_{1}\left(t-\tau_{1}\right) \\
& -\left(1-\rho_{1}\right) e^{-\alpha_{2} \tau_{2}} d_{1} x_{1}\left(t-\tau_{2}\right) \\
& -\rho_{2} e^{-\alpha_{3} \tau_{3}} d_{2} x_{2}\left(t-\tau_{3}\right)-\left(1-\rho_{2}\right) e^{-\alpha_{4} \tau_{4}} d_{2} x_{2}\left(t-\tau_{4}\right)-a y_{1}(t)-a y_{2}(t) \\
& -\frac{\mu}{2} u_{1}(t)-\frac{\mu}{2} u_{2}(t)-\frac{\mu b}{2 c} z(t) \\
\leq & \lambda_{1}+\lambda_{2}-\delta_{1} U_{1}(t) .
\end{aligned}
$$

Then $\limsup _{t \rightarrow \infty} x_{i}(t) \leq M_{1}, \quad \lim \sup _{t \rightarrow \infty} y_{i}(t) \leq M_{1}, \quad \limsup \sup _{t \rightarrow \infty} u_{i}(t) \leq M_{1}$, $\lim \sup _{t \rightarrow \infty} z(t) \leq M_{2}$ and $\limsup \sup _{t \rightarrow \infty} v(t) \leq M_{3}$, where $M_{1}, M_{2}$ and $M_{3}$ are defined in the previous section.

\subsection{Steady states}

The basic reproduction number $R_{0}$ for system (25)-(32) is as follows:

$$
R_{0}=\frac{\delta k G}{\mu r(a+\delta)}\left(\xi_{1} \beta_{1} x_{1}^{0}+\xi_{2} \beta_{2} x_{2}^{0}\right)
$$

where

$$
\xi_{1}=\rho_{1} H_{1}+\frac{(a+\delta)}{\delta}\left(1-\rho_{1}\right) H_{2}, \quad \xi_{2}=\rho_{2} H_{3}+\frac{(a+\delta)}{\delta}\left(1-\rho_{2}\right) H_{4} .
$$


Lemma 2 For system (25)-(32) (i) if $R_{0} \leq 1$, then there exists an infection-free steady state $E_{0}$, and (ii) if $R_{0}>1$, then there exist two steady states, $E_{0}$ and a chronic steady state $E^{*}$.

Proof The steady states of model (25)-(32) satisfy:

$$
\begin{aligned}
& \lambda_{1}-d_{1} x_{1}-\beta_{1} x_{1} v=0, \\
& \rho_{1} \beta_{1} H_{1} x_{1} v-a y_{1}-\delta y_{1}=0, \\
& \left(1-\rho_{1}\right) \beta_{1} H_{2} x_{1} v+\delta y_{1}-p u_{1} z-\mu u_{1}=0, \\
& \lambda_{2}-d_{2} x_{2}-\beta_{2} x_{2} v=0, \\
& \rho_{2} \beta_{2} H_{3} x_{2} v-a y_{2}-\delta y_{2}=0, \\
& \left(1-\rho_{2}\right) \beta_{2} H_{4} x_{2} v+\delta y_{2}-p u_{2} z-\mu u_{2}=0, \\
& k G\left(u_{1}+u_{2}\right)-r v=0, \\
& c\left(u_{1}+u_{2}\right)-m\left(u_{1}+u_{2}\right) z-b z=0 .
\end{aligned}
$$

We find that system (25)-(32) admits an infection-free steady state $E_{0}=\left(x_{1}^{0}, 0,0, x_{2}^{0}, 0,0\right.$, $0,0)$ where $x_{1}^{0}=\frac{\lambda_{1}}{d_{1}}, x_{2}^{0}=\frac{\lambda_{2}}{d_{2}}$. In addition, from Eqs. (33)-(40) we get

$$
A_{2} v^{3}+B_{2} v^{2}+C_{2} v+D_{2}=0
$$

where

$$
\begin{aligned}
& A_{2}=\beta_{1} \beta_{2}(\mu m+p c) r^{2}, \\
& B_{2}=\beta_{1} \beta_{2} \mu r b G k+\left(\beta_{1} d_{2}+\beta_{2} d_{1}\right)(\mu m+p c) r^{2}-\frac{\beta_{1} \beta_{2} m r \delta G k}{a+\delta}\left(\lambda_{1} \xi_{1}+\lambda_{2} \xi_{2}\right), \\
& C_{2}=\mu r b G k\left(\beta_{1} d_{2}+\beta_{2} d_{1}\right)+\mu m r^{2} d_{1} d_{2}\left(1-R_{0}\right)+p c r^{2} d_{1} d_{2}-\frac{\beta_{1} \beta_{2} \delta b G^{2} k^{2}}{a+\delta}\left(\lambda_{1} \xi_{1}+\lambda_{2} \xi_{2}\right), \\
& D_{2}=\mu r b k d_{1} d_{2} G\left(1-R_{0}\right) .
\end{aligned}
$$

Let

$$
\Psi_{2}(v)=A_{2} v^{3}+B_{2} v^{2}+C_{2} v+D_{2}=0
$$

If $R_{0}>1$, then $\Psi_{2}(0)=D_{2}<0$; moreover, $\lim _{v \rightarrow \infty} \Psi_{2}(v)=\infty$. Then there exits $v^{*} \in(0, \infty)$ such that $\Psi_{2}\left(v^{*}\right)=0$. Therefore, a chronic steady state $E^{*}=\left(x_{1}^{*}, y_{1}^{*}, u_{1}^{*}, x_{2}^{*}, y_{2}^{*}, u_{2}^{*}, v^{*}, z^{*}\right)$ exists if $R_{0}>1$, where

$$
\begin{aligned}
& x_{i}^{*}=\frac{\lambda_{i}}{d_{i}+\beta_{i} v^{*}}, \quad i=1,2, \\
& y_{1}^{*}=\frac{\rho_{1} \beta_{1} H_{1} x_{1}^{*} v^{*}}{a+\delta}, \quad y_{2}^{*}=\frac{\rho_{2} \beta_{2} H_{3} x_{2}^{*} v^{*}}{a+\delta}, \\
& u_{i}^{*}=\frac{\xi_{i} \beta_{i} x_{i}^{*} v^{*} \delta}{\left(\mu+p z^{*}\right)(a+\delta)}, \quad \sum_{i=1}^{2} u_{i}^{*}=\frac{r v^{*}}{G k}, \\
& z^{*}=\frac{c r v^{*}}{b k G+m r v^{*}} .
\end{aligned}
$$




\subsection{Global stability}

Theorem 3 If $R_{0} \leq 1$, then $E_{0}$ for system (25)-(32) is globally asymptotically stable.

Proof Define

$$
\begin{aligned}
V_{1}= & \sum_{i=1}^{2}\left[\xi_{i} x_{i}^{0} f\left(\frac{x_{i}}{x_{i}^{0}}\right)+y_{i}+\frac{a+\delta}{\delta} u_{i}\right]+\frac{\mu(a+\delta)}{\delta G k} v+\frac{p(a+\delta)}{2 \delta c} z^{2} \\
& +\rho_{1} \beta_{1} \int_{0}^{s_{1}} h_{1}(\tau) e^{-m_{1} \tau} \int_{0}^{\tau} x_{1}(t-\vartheta) v(t-\vartheta) d \vartheta d \tau \\
& +\frac{(a+\delta)}{\delta}\left(1-\rho_{1}\right) \int_{0}^{s_{2}} h_{2}(\tau) e^{-m_{2} \tau} \int_{0}^{\tau} \beta_{1} x_{1}(t-\vartheta) v(t-\vartheta) d \vartheta d \tau \\
& +\rho_{2} \beta_{2} \int_{0}^{s_{3}} h_{3}(\tau) e^{-m_{3} \tau} \int_{0}^{\tau} x_{2}(t-\vartheta) v(t-\vartheta) d \vartheta d \tau \\
& +\frac{(a+\delta)}{\delta}\left(1-\rho_{2}\right) \beta_{2} \int_{0}^{s_{4}} h_{4}(\tau) e^{-m_{4} \tau} \int_{0}^{\tau} x_{2}(t-\vartheta) v(t-\vartheta) d \vartheta d \tau \\
& +\frac{\mu(a+\delta)}{\delta G} \int_{0}^{s_{5}} g(\tau) e^{-n \tau} \int_{0}^{\tau}\left[u_{1}(t-\vartheta)+u_{2}(t-\vartheta)\right] d \vartheta d \tau .
\end{aligned}
$$

Calculating $\dot{V}_{1}$ along system (25)-(32), we obtain

$$
\begin{aligned}
\dot{V}_{1}= & \sum_{i=1}^{2}\left[\xi_{i}\left(1-\frac{x_{i}^{0}}{x_{i}}\right) \dot{x}_{i}+\dot{y}_{i}+\frac{a+\delta}{\delta} \dot{u}_{i}\right]+\frac{\mu(a+\delta)}{\delta G k} \dot{v}+\frac{p(a+\delta)}{\delta c} z \dot{z} \\
& +\rho_{1} \beta_{1} \int_{0}^{s_{1}} h_{1}(\tau) e^{-m_{1} \tau}\left[x_{1}(t) v(t)-x_{1}(t-\tau) v(t-\tau)\right] d \tau \\
& +\frac{(a+\delta)\left(1-\rho_{1}\right)}{\delta} \beta_{1} \int_{0}^{s_{2}} h_{2}(\tau) e^{-m_{2} \tau}\left[x_{1}(t) v(t)-x_{1}(t-\tau) v(t-\tau)\right] d \tau \\
& +\rho_{2} \beta_{2} \int_{0}^{s_{3}} h_{3}(\tau) e^{-m_{3} \tau}\left[x_{2} v-x_{2}(t-\tau) v(t-\tau)\right] d \tau \\
& +\frac{(a+\delta)\left(1-\rho_{2}\right)}{\delta} \beta_{2} \int_{0}^{s_{4}} h_{4}(\tau) e^{-m_{4} \tau}\left[x_{2} v-x_{2}(t-\tau) v(t-\tau)\right] d \tau \\
& +\frac{\mu(a+\delta)}{\delta G} \int_{0}^{s_{5}} g(\tau) e^{-n \tau}\left[u_{1}+u_{2}-u_{1}(t-\tau)-u_{2}(t-\tau)\right] d \tau .
\end{aligned}
$$

Since $\lambda_{i}=d_{i} x_{i}^{0}$, we get

$$
\begin{aligned}
\dot{V}_{1}= & \sum_{i=1}^{2}\left[\xi_{i}\left(1-\frac{x_{i}^{0}}{x_{i}}\right)\left(d_{i} x_{i}^{0}-d_{i} x_{i}\right)-\xi_{i} \beta_{i} x_{i} v\left(1-\frac{x_{i}^{0}}{x_{i}}\right)+\xi_{i} \beta_{i} x_{i} v\right]-\frac{\mu(a+\delta)}{\delta G k} r v \\
& -\frac{p m(a+\delta)}{\delta c}\left(u_{1}+u_{2}\right) z^{2}-\frac{p b(a+\delta)}{\delta c} z^{2} \\
= & \sum_{i=1}^{2} \frac{-d_{i} \xi_{i}\left(x_{i}-x_{i}^{0}\right)^{2}}{x_{i}}+\frac{\mu r(a+\delta)}{\delta G k}\left(R_{0}-1\right) v-\frac{p m(a+\delta)}{\delta} \sum_{i=1}^{2} u_{i} z^{2}-\frac{p b(a+\delta)}{\delta c} z^{2} .
\end{aligned}
$$

Similar to the proof of Theorem $1, E_{0}$ is globally asymptotically stable if $R_{0} \leq 1$.

Theorem 4 If $R_{0}>1$, then $E^{*}$ for system (25)-(32) is globally asymptotically stable. 
Proof Define

$$
\begin{aligned}
V_{2}= & \sum_{i=1}^{2}\left[\xi_{i} x_{i}^{*} f\left(\frac{x_{i}}{x_{i}^{*}}\right)+y_{i}^{*} f\left(\frac{y_{i}}{y_{i}^{*}}\right)+\frac{(a+\delta)}{\delta} u_{i}^{*} f\left(\frac{u_{i}}{u_{i}^{*}}\right)\right]+\frac{(a+\delta)\left(\mu+p z^{*}\right)}{\delta G k} v^{*} f\left(\frac{v}{v^{*}}\right) \\
& +\frac{p(a+\delta)}{2 \delta\left(c-m z^{*}\right)}\left(z-z^{*}\right)^{2} \\
& +\rho_{1} \beta_{1} x_{1}^{*} v^{*} \int_{0}^{s_{1}} h_{1}(\tau) e^{-m_{1} \tau} \int_{0}^{\tau} f\left(\frac{x_{1}(t-\vartheta) v(t-\vartheta)}{x_{1}^{*} v^{*}}\right) d \vartheta d \tau \\
& +\frac{(a+\delta)}{\delta}\left(1-\rho_{1}\right) \beta_{1} x_{1}^{*} v^{*} \int_{0}^{s_{2}} h_{2}(\tau) e^{-m_{2} \tau} \int_{0}^{\tau} f\left(\frac{x_{1}(t-\vartheta) v(t-\vartheta)}{x_{1}^{*} v^{*}}\right) d \vartheta d \tau \\
& +\rho_{2} \beta_{2} x_{2}^{*} v^{*} \int_{0}^{s_{3}} h_{3}(\tau) e^{-m_{3} \tau} \int_{0}^{\tau} f\left(\frac{x_{2}(t-\vartheta) v(t-\vartheta)}{x_{2}^{*} v^{*}}\right) d \vartheta d \tau \\
& +\frac{(a+\delta)}{\delta}\left(1-\rho_{2}\right) \beta_{2} x_{2}^{*} v^{*} \int_{0}^{s_{4}} h_{4}(\tau) e^{-m_{4} \tau} \int_{0}^{\tau} f\left(\frac{x_{2}(t-\vartheta) v(t-\vartheta)}{x_{2}^{*} v^{*}}\right) d \vartheta d \tau \\
& +\frac{(a+\delta)\left(\mu+p z^{*}\right)}{\delta G} \int_{0}^{s_{5}} g(\tau) e^{-n \tau} \int_{0}^{\tau}\left[u_{1}^{*} f\left(\frac{u_{1}(t-\vartheta)}{u_{1}^{*}}\right)+u_{2}^{*} f\left(\frac{u_{2}(t-\vartheta)}{u_{2}^{*}}\right)\right] d \vartheta d \tau .
\end{aligned}
$$

It is seen that function $V_{2}$ is positive definite. The time derivative of $V_{2}$ is given by the following:

$$
\begin{aligned}
& \dot{V}_{2}=\sum_{i=1}^{2}\left[\xi_{i}\left(1-\frac{x_{i}^{*}}{x_{i}}\right) \dot{x}_{i}+\left(1-\frac{y_{i}^{*}}{y_{i}}\right) \dot{y}_{i}+\frac{(a+\delta)}{\delta}\left(1-\frac{u_{i}^{*}}{u_{i}}\right) \dot{u}_{i}\right] \\
& +\frac{(a+\delta)\left(\mu+p z^{*}\right)}{\delta G k}\left(1-\frac{v^{*}}{v}\right) \dot{v} \\
& +\frac{p(a+\delta)}{\delta\left(c-m z^{*}\right)}\left(z-z^{*}\right) \dot{z}+\rho_{1} \beta_{1} x_{1}^{*} v^{*} \int_{0}^{s_{1}} h_{1}(\tau) e^{-m_{1} \tau}\left[\frac{x_{1} v}{x_{1}^{*} v^{*}}-\frac{x_{1}(t-\tau) v(t-\tau)}{x_{1}^{*} v^{*}}\right. \\
& \left.+\ln \frac{x_{1}(t-\tau) v(t-\tau)}{x_{1} v}\right] d \tau \\
& +\frac{(a+\delta)}{\delta}\left(1-\rho_{1}\right) \beta_{1} x_{1}^{*} v^{*} \int_{0}^{s_{2}} h_{2}(\tau) e^{-m_{2} \tau}\left[\frac{x_{1} v}{x_{1}^{*} v^{*}}-\frac{x_{1}(t-\tau) v(t-\tau)}{x_{1}^{*} v^{*}}\right. \\
& \left.+\ln \frac{x_{1}(t-\tau) v(t-\tau)}{x_{1} v}\right] d \tau \\
& +\rho_{2} \beta_{2} x_{2}^{*} v^{*} \int_{0}^{s_{3}} h_{3}(\tau) e^{-m_{3} \tau}\left[\frac{x_{2} v}{x_{2}^{*} v^{*}}-\frac{x_{2}(t-\tau) v(t-\tau)}{x_{2}^{*} v^{*}}+\ln \frac{x_{2}(t-\tau) v(t-\tau)}{x_{2} v}\right] d \tau \\
& +\frac{(a+\delta)}{\delta}\left(1-\rho_{2}\right) \beta_{2} x_{2}^{*} v^{*} \int_{0}^{s_{4}} h_{4}(\tau) e^{-m_{4} \tau}\left[\frac{x_{2} v}{x_{2}^{*} v^{*}}-\frac{x_{2}(t-\tau) v(t-\tau)}{x_{2}^{*} v^{*}}\right. \\
& \left.+\ln \frac{x_{2}(t-\tau) v(t-\tau)}{x_{2} v}\right] d \tau \\
& +\frac{(a+\delta)\left(\mu+p z^{*}\right)}{\delta G} u_{1}^{*} \int_{0}^{s_{5}} g(\tau) e^{-n \tau}\left[\frac{u_{1}}{u_{1}^{*}}-\frac{u_{1}(t-\tau)}{u_{1}^{*}}+\ln \frac{u_{1}(t-\tau)}{u_{1}}\right] d \tau \\
& +\frac{(a+\delta)\left(\mu+p z^{*}\right)}{\delta G} u_{2}^{*} \int_{0}^{s_{5}} g(\tau) e^{-n \tau}\left[\frac{u_{2}}{u_{2}^{*}}-\frac{u_{2}(t-\tau)}{u_{2}^{*}}+\ln \frac{u_{2}(t-\tau)}{u_{2}}\right] d \tau \text {. }
\end{aligned}
$$


Using chronic steady state conditions

$$
\begin{aligned}
& \lambda_{i}=d_{i} x_{i}^{*}+\beta_{i} x_{i}^{*} v^{*}, \quad(a+\delta) y_{1}^{*}=\rho_{1} \beta_{1} H_{1} x_{1}^{*} v^{*}, \\
& (a+\delta) y_{2}^{*}=\rho_{2} \beta_{2} H_{3} x_{2}^{*} v^{*}, \quad(a+\delta)\left(\mu+p z^{*}\right) u_{i}^{*}=\xi_{i} \beta_{i} \delta x_{i}^{*} v^{*}, \\
& r v^{*}=k G\left(u_{1}^{*}+u_{2}^{*}\right), \quad 0=c\left(u_{1}^{*}+u_{2}^{*}\right)-m\left(u_{1}^{*}+u_{2}^{*}\right) z^{*}-b z^{*},
\end{aligned}
$$

we can simplify:

$$
\begin{aligned}
& \frac{p}{\left(c-m z^{*}\right)}\left(z-z^{*}\right) \dot{z} \\
& =\frac{p(a+\delta)\left(z-z^{*}\right)}{\delta\left(c-m z^{*}\right)}\left(c\left(u_{1}+u_{2}\right)-m\left(u_{1}+u_{2}\right) z-b z\right) \\
& =p\left[\sum_{i=1}^{2} u_{i}^{*} z^{*}-\sum_{i=1}^{2} u_{i}^{*} z-\sum_{i=1}^{2} u_{i} z^{*}+\sum_{i=1}^{2} u_{i} z-\frac{\left[b+m \sum_{i=1}^{2} u_{i}\right]}{\left(c-m z^{*}\right)}\left(z-z^{*}\right)^{2}\right] .
\end{aligned}
$$

The following relations will be used:

$$
\begin{aligned}
& \ln \left(\frac{x_{1}(t-\tau) v(t-\tau)}{x_{1} v}\right)=\ln \left(\frac{x_{1}^{*} v^{*} y_{1}}{x_{1} v y_{1}^{*}}\right)+\ln \left(\frac{y_{1}^{*} x_{1}(t-\tau) v(t-\tau)}{y_{1} x_{1}^{*} v^{*}}\right), \\
& \ln \left(\frac{x_{1}(t-\tau) v(t-\tau)}{x_{1} v}\right)=\ln \left(\frac{x_{1}^{*} v^{*} u_{1}}{x_{1} v u_{1}^{*}}\right)+\ln \frac{u_{1}^{*} x_{1}(t-\tau) v(t-\tau)}{u_{1} x_{1}^{*} v^{*}}, \\
& \ln \left(\frac{u_{i}(t-\tau)}{u_{i}}\right)=\ln \left(\frac{v u_{i}^{*}}{v^{*} u_{i}}\right)+\ln \left(\frac{u_{i}(t-\tau) v^{*}}{u_{i}^{*} v}\right) .
\end{aligned}
$$

Now using the last relations with steady state conditions and Eq. (42), we can rewrite

$$
\begin{aligned}
\dot{V}_{2}(t)= & \sum_{i=1}^{2}\left[\frac{-\xi_{i} d_{i}\left(x_{i}-x_{i}^{*}\right)^{2}}{x_{i}}+\xi_{i} \beta_{i} x_{i}^{*} v^{*}\left(1-\frac{x_{i}^{*}}{x_{i}}\right)\right. \\
& -\frac{\xi_{i} \beta_{i} x_{i}^{*} v^{*}}{G} \int_{0}^{s_{5}} g(\tau) e^{-n \tau} f\left(\frac{v^{*} u_{i}(t-\tau)}{v u_{i}^{*}}\right) d \tau \\
& \left.+\frac{\xi_{i} \beta_{i} x_{i}^{*} v^{*}}{G} \int_{0}^{s_{5}} g(\tau) e^{-n \tau} \ln \frac{v u_{i}^{*}}{v^{*} u_{i}} d \tau\right]-\rho_{1} \beta_{1} H_{1} x_{1}^{*} v^{*}\left(\frac{y_{1} u_{1}^{*}}{y_{1}^{*} u_{1}}-1\right) \\
& -\rho_{2} \beta_{2} H_{3} x_{2}^{*} v^{*}\left(\frac{y_{2} u_{2}^{*}}{y_{2}^{*} u_{2}}-1\right) \\
& -\rho_{1} \beta_{1} x_{1}^{*} v^{*} \int_{0}^{s_{1}} h_{1}(\tau) e^{-m_{1} \tau} f\left(\frac{y_{1}^{*} x_{1}(t-\tau) v(t-\tau)}{y_{1} x_{1}^{*} v^{*}}\right) d \tau \\
& +\rho_{1} \beta_{1} x_{1}^{*} v^{*} \int_{0}^{s_{1}} h_{1}(\tau) e^{-m_{1} \tau} \ln \frac{y_{1} x_{1}^{*} v^{*}}{y_{1}^{*} x_{1} v} d \tau \\
& -\rho_{2} \beta_{2} x_{2}^{*} v^{*} \int_{0}^{s_{3}} h_{3}(\tau) e^{-m_{3} \tau} f\left(\frac{y_{2}^{*} x_{2}(t-\tau) v(t-\tau)}{y_{2} x_{2}^{*} v^{*}}\right) d \tau \\
& +\rho_{2} \beta_{2} x_{2}^{*} v^{*} \int_{0}^{s_{3}} h_{3}(\tau) e^{-m_{3} \tau} \ln \frac{y_{2} x_{2}^{*} v^{*}}{y_{2}^{*} x_{2} v} d \tau \\
& +\frac{a+\delta}{\delta}\left(1-\rho_{1}\right) \beta_{1} x_{1}^{*} v^{*} \int_{0}^{s_{2}} h_{2}(\tau) e^{-m_{2} \tau} \ln \frac{u_{1} x_{1}^{*} v^{*}}{u_{1}^{*} x_{1} v} d \tau
\end{aligned}
$$




$$
\begin{aligned}
& +\frac{a+\delta}{\delta} \rho_{2} \beta_{2} x_{2}^{*} v^{*} \int_{0}^{s_{4}} h_{4}(\tau) e^{-m_{4} \tau} \ln \frac{u_{2} x_{2}^{*} v^{*}}{u_{2}^{*} x_{2} v} d \tau \\
& -\frac{a+\delta}{\delta}\left(1-\rho_{1}\right) \beta_{1} x_{1}^{*} v^{*} \int_{0}^{s_{2}} h_{2}(\tau) e^{-m_{2} \tau} f\left(\frac{u_{1}^{*} x_{1}(t-\tau) \nu(t-\tau)}{u_{1} x_{1}^{*} v^{*}}\right) d \tau \\
& -\frac{a+\delta}{\delta}\left(1-\rho_{2}\right) \beta_{2} x_{2}^{*} v^{*} \int_{0}^{s_{4}} h_{4}(\tau) e^{-m_{4} \tau} f\left(\frac{u_{2}^{*} x_{2}(t-\tau) v(t-\tau)}{u_{2} x_{2}^{*} v^{*}}\right) d \tau \\
& -\frac{p(a+\delta)\left[b+m\left(u_{1}+u_{2}\right)\right]}{\delta\left(c-m z^{*}\right)}\left(z-z^{*}\right)^{2} .
\end{aligned}
$$

Finally, we obtain

$$
\begin{aligned}
\dot{V}_{2}(t)= & -\sum_{i=1}^{2}\left[\frac{\xi_{i} d_{i}\left(x_{i}-x_{i}^{*}\right)^{2}}{x_{i}}+\xi_{i} \beta_{i} x_{i}^{*} \nu^{*} f\left(\frac{x_{i}^{*}}{x_{i}}\right)\right. \\
& \left.+\frac{\xi_{i} \beta_{i} x_{i}^{*} v^{*}}{G} \int_{0}^{s_{5}} g(\tau) e^{-n \tau} f\left(\frac{v^{*} u_{i}(t-\tau)}{v u_{i}^{*}}\right) d \tau\right] \\
& -\rho_{1} \beta_{1} H_{1} x_{1}^{*} v^{*} f\left(\frac{y_{1} u_{1}^{*}}{y_{1}^{*} u_{1}}\right)-\rho_{1} \beta_{1} x_{1}^{*} v^{*} \int_{0}^{s_{1}} h_{1}(\tau) e^{-m_{1} \tau} f\left(\frac{y_{1}^{*} x_{1}(t-\tau) v(t-\tau)}{y_{1} x_{1}^{*} v^{*}}\right) d \tau \\
& -\rho_{2} \beta_{2} H_{3} x_{2}^{*} v^{*} f\left(\frac{y_{2} u_{2}^{*}}{y_{2}^{*} u_{2}}\right)-\rho_{2} \beta_{2} x_{2}^{*} v^{*} \int_{0}^{s_{3}} h_{3}(\tau) e^{-m_{3} \tau} f\left(\frac{y_{2}^{*} x_{2}(t-\tau) v(t-\tau)}{y_{2} x_{2}^{*} v^{*}}\right) d \tau \\
& -\frac{a+\delta}{\delta}\left(1-\rho_{1}\right) \beta_{1} x_{1}^{*} v^{*} \int_{0}^{s_{2}} h_{2}(\tau) e^{-m_{2} \tau} f\left(\frac{u_{1}^{*} x_{1}(t-\tau) v(t-\tau)}{u_{1} x_{1}^{*} v^{*}}\right) d \tau \\
& -\frac{a+\delta}{\delta}\left(1-\rho_{2}\right) \beta_{2} x_{2}^{*} v^{*} \int_{0}^{s_{4}} h_{4}(\tau) e^{-m_{4} \tau} f\left(\frac{u_{2}^{*} x_{2}(t-\tau) v(t-\tau)}{u_{2} x_{2}^{*} v^{*}}\right) d \tau \\
& -\frac{p(a+\delta)\left[b+m\left(u_{1}+u_{2}\right)\right]}{\delta\left(c-m z^{*}\right)}\left(z-z^{*}\right)^{2} .
\end{aligned}
$$

Applying LaSalle's invariance principle to the last equation, we get $\dot{V}_{2} \leq 0$, leading to the global asymptotic stability of the chronic steady state $E^{*}$.

\section{Numerical simulations}

In this section, we perform numerical simulation for model (6)-(13) with values of the parameters given in Table 1. Let us consider for simplicity that $\tau_{1}=\tau_{2}=\tau_{3}=\tau_{4}=\tau_{5}=\tau_{c}$, and chose the initial conditions as:

(IC1) $\omega_{1}(\eta)=700, \omega_{2}(\eta)=20, \omega_{3}(\eta)=15, \omega_{4}(\eta)=15, \omega_{5}(\eta)=0.0005, \omega_{6}(\eta)=$ $0.0005, \omega_{7}(\eta)=15$ and $\omega_{8}(\eta)=1.5$;

(IC2) $\omega_{1}(\eta)=500, \omega_{2}(\eta)=15, \omega_{3}(\eta)=10, \omega_{4}(\eta)=10, \omega_{5}(\eta)=0.001, \omega_{6}(\eta)=$ $0.001, \omega_{7}(\eta)=12$ and $\omega_{8}(\eta)=1$

(IC3) $\omega_{1}(\eta)=300, \omega_{2}(\eta)=5, \omega_{3}(\eta)=5, \omega_{4}(\eta)=1, \omega_{5}(\eta)=0.3, \omega_{6}(\eta)=0.3, \omega_{7}(\eta)=8$ and $\omega_{8}(\eta)=0.5, \eta \in\left[-\max \left\{\tau_{1}, \tau_{2}, \tau_{3}, \tau_{4}, \tau_{5}\right\}, 0\right]$.

\section{Case I. Stability of the steady states}

Let $\tau_{c}=0.7$ and $\rho_{1}=\rho_{2}=0.5$. We have two subcases shown in Fig. 1 :

(a) if $\beta_{1}=2.4 \times 10^{-5}$ and $\beta_{2}=2 \times 10^{-5}$, then $R_{0}=0.0264<1$. It follows that $E_{0}$ is globally asymptotically stable. In this case the concentration of the uninfected cells is increasing and returns to its normal value $\lambda_{i} / d_{i}$, while the concentrations of infected cells, HIV particles and CTLs are decaying and approach zero. 
Table 1 The parameter values of model (6)-(13)

\begin{tabular}{llll}
\hline Parameter & Value & Parameter & Value \\
\hline$\lambda_{1}$ & 10 & $\lambda_{2}$ & 0.03198 \\
$d_{1}$ & 0.01 & $d_{2}$ & 0.01 \\
$\beta_{1}$ & varied & $\beta_{2}$ & varied \\
$\rho_{1}$ & varied & $\rho_{2}$ & varied \\
$a$ & 0.1 & $\alpha_{1}$ & 1 \\
$p$ & 0.04 & $\alpha_{2}$ & 1 \\
$k$ & 6 & $\alpha_{3}$ & 1 \\
$r$ & 3 & $\alpha_{4}$ & 1 \\
$c$ & 0.025 & $\alpha_{5}$ & 1 \\
$b$ & 0.2 & $\delta$ & 0.05 \\
$\mu$ & 0.3 & $m$ & 0.005 \\
\hline
\end{tabular}

(b) if $\beta_{1}=0.002$ and $\beta_{2}=0.001$ then $R_{0}=2.1955>1$. Therefore, $E^{*}$ exists and is globally asymptotically stable. In this case the concentration of the uninfected cells is decreasing, while the concentrations of infected cells, HIV particles and CTLs are increasing. In this situation HIV infection will be chronic.

From the above, one can see that the infection rate parameters $\beta_{1}$ and $\beta_{2}$ have significant effects on stabilizing the infection-free steady state. This is because $R_{0}$ can be decreased by decreasing the values of $\beta_{1}$ and $\beta_{2}$. The values of the parameters $\beta_{1}$ and $\beta_{2}$ can be reduced when an HIV infected patient is treated by reverse transcriptase inhibitor (RTI) drugs which prevent HIV from infecting the uninfected target cells. When incorporating the RTI drugs into the HIV dynamics model, the parameters $\beta_{1}$ and $\beta_{2}$ will be replaced by $(1-\epsilon) \beta_{1}$ and $(1-\epsilon) \beta_{2}$, respectively, where $\epsilon$ is the drug efficacy with $0 \leq \epsilon<1$. In this case, the basic reproduction number is given by

$$
R_{0}(\epsilon)=\frac{(1-\epsilon) \delta k}{\mu r(a+\delta)}\left(\gamma_{1} \beta_{1} x_{1}^{0}+\gamma_{2} \beta_{2} x_{2}^{0}\right) .
$$

Therefore, one can find the minimum drug efficacy $\epsilon_{m}$, which makes $R_{0}(\epsilon) \leq 1$ and then stabilizes the system around the infection-free steady state $E_{0}$, as:

$$
\epsilon_{m}=\max \left\{1-\frac{\mu r(a+\delta)}{\delta k\left(\gamma_{1} \beta_{1} x_{1}^{0}+\gamma_{2} \beta_{2} x_{2}^{0}\right)}, 0\right\} .
$$

\section{Case II. Effect of the time delay on the HIV dynamics}

In this case, we fix the parameters $\beta_{1}=0.002, \beta_{2}=0.001$ and $\rho_{1}=\rho_{2}=0.5$. We consider initial conditions IC1. Figure 2 shows that the stability of the steady states has been changed by changing the values of the delay parameter $\tau_{c}$. It can be seen that as $\tau_{c}$ is increased the concentration of the uninfected cells is increased, while the concentrations of infected cells, free HIV particles and CTLs are decreased. From Fig. 2 we can see that, in case of smaller values of $\tau_{c}$, trajectories of the system will converge to $E^{*}$. If the value of $\tau_{c}$ is increased, trajectories will converge to $E^{*}$ and finally approach $E_{0}$. Using the values of the parameters given in Table 1, we have the following:

(i) if $0.0 \leq \tau_{c}<1.0932$, then $E^{*}$ exists and is globally asymptotically stable;

(ii) if $\tau_{c} \geq 1.0932$, then $E_{0}$ is globally asymptotically stable.

This result support the results of Theorems 1 and 2 .

From a biological point of view, the intracellular delay plays a similar role as an antiviral treatment in eliminating the virus. We observe that sufficiently large delay suppresses viral 


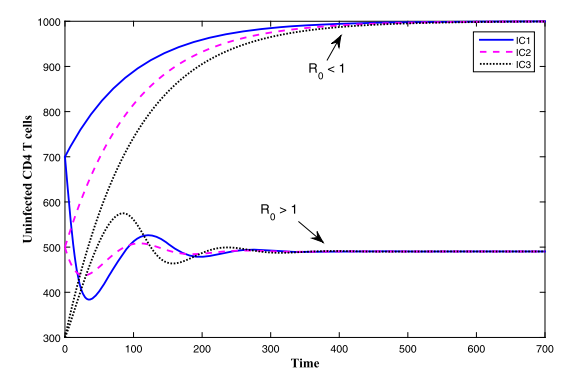

(a) The concentration of uninfected CD4 T cells

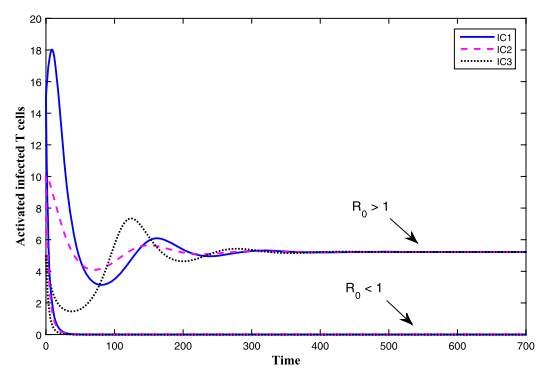

(c) The concentration of actively infected CD4 $\mathrm{T}$ cells

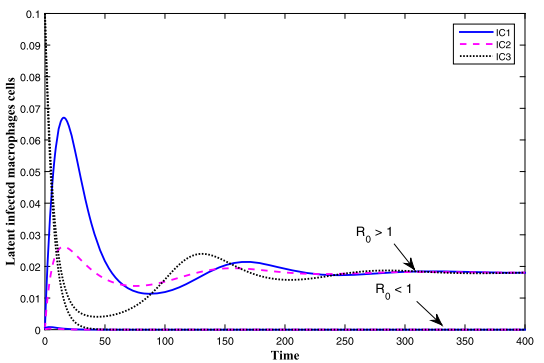

(e) The conc. of latently infected macrophages cells

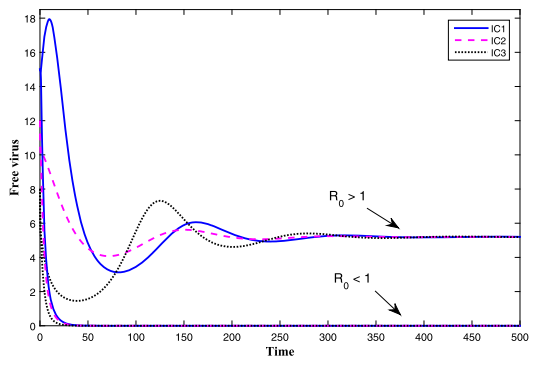

(g) The concentration of free virus

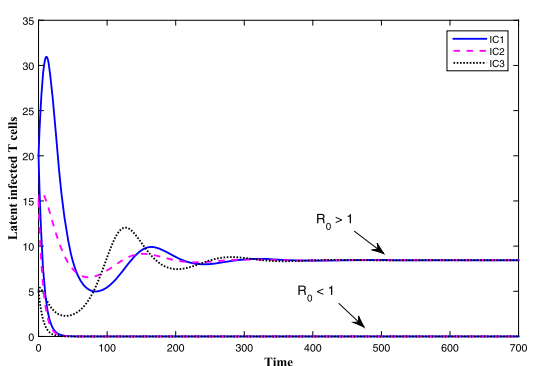

(b) The concentration of latently infected CD4 T cells

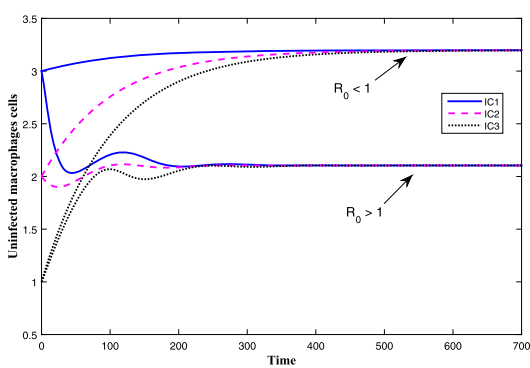

(d) The conc. of uninfected macrophages cells

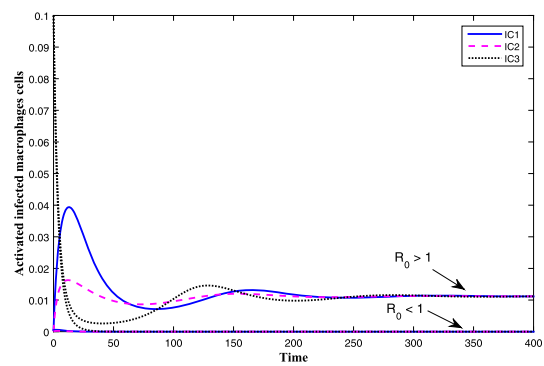

(f) The conc. of actively infected macrophages cells

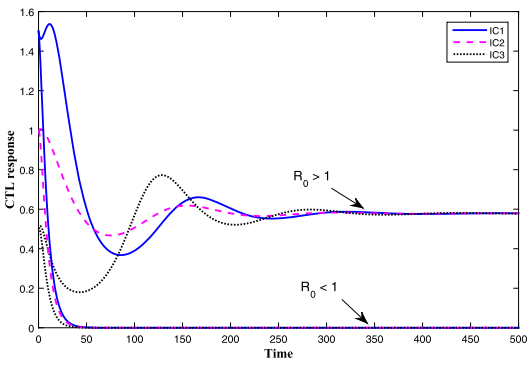

(h) The concentration of CTL immune response

Figure 1 The evolution of model (6)-(13) with varied infection rates

replication and clears the virus from the body. This gives us some suggestions on new drugs to prolong the increase of the intracellular delay period.

Case III. Effect of latency on the dynamical behavior of the system

In this case, we show the HIV dynamics for different values of $\rho_{i}$, the fraction of uninfected cells that become latently infected cells. We take $\beta_{1}=0.002, \beta_{2}=0.001, \tau_{c}=0.8$ and the initial conditions IC1. For simplicity we let $\rho_{1}=\rho_{2}=\rho_{c}$. Figure 4 shows the effect 


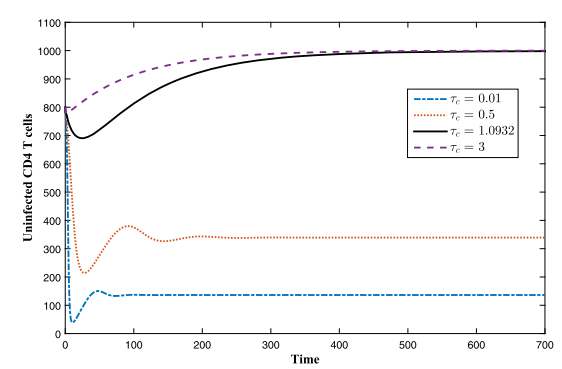

(a) The concentration of uninfected CD4 T cells

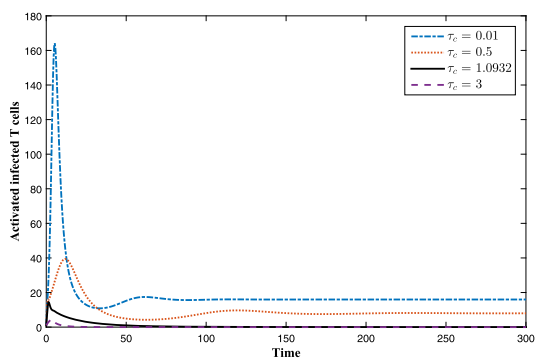

(c) The concentration of actively infected CD4 T cells

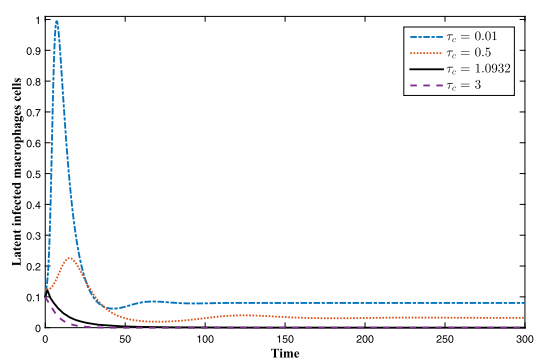

(e) The conc. of latently infected macrophages cells

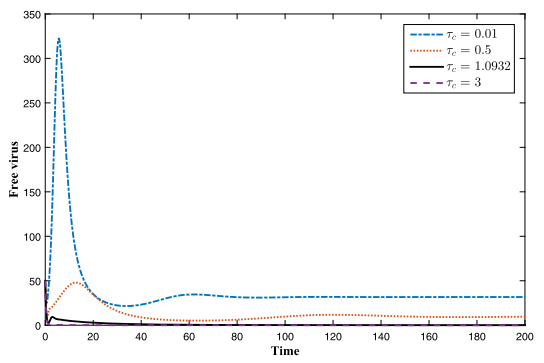

(g) The concentration of free virus

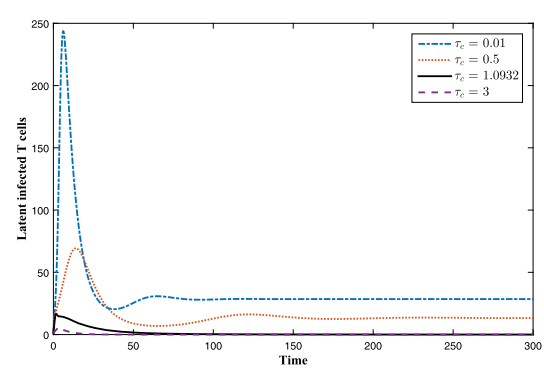

(b) The concentration of latently infected CD4 T cells

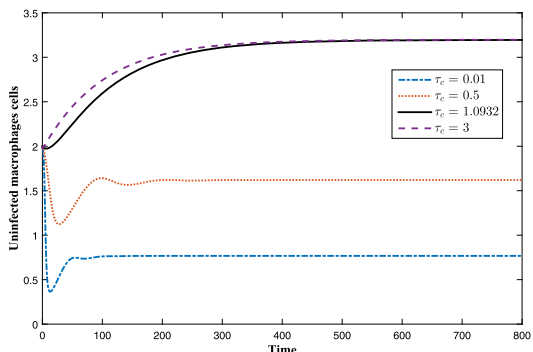

(d) The conc. of uninfected macrophages cells

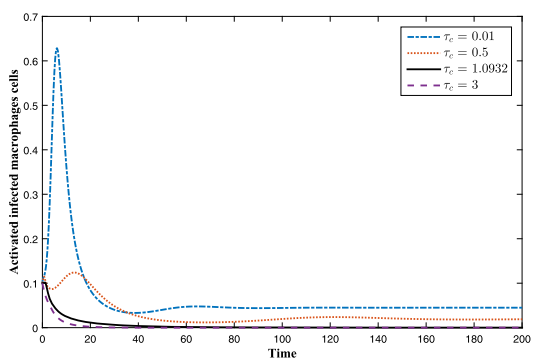

(f) The conc. of actively infected macrophages cells

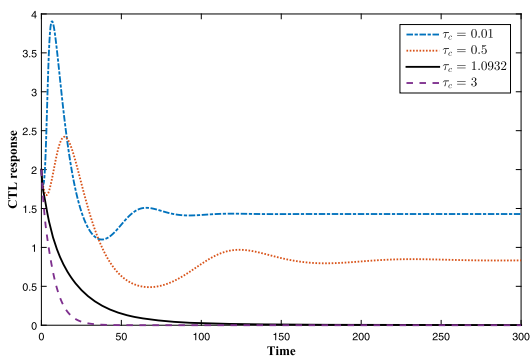

(h) The concentration of CTL immune response

Figure 2 The evolution of model (6)-(13) with varied $\tau_{c}$

of $\rho_{c}$ on the the evolution of system states. When $\rho_{c}$ increases, we observe an increase in the concentration of the latently infected $\mathrm{CD} 4^{+} \mathrm{T}$ cells and macrophages. This means that the reservoirs of these cells are enlarged, which promotes an increase in the amount of virus that escapes treatment [18]. Subsequently, after activation of the latently infected cells, new HIV will be produced and released into the blood stream [55]. From Fig. 4 we can see that, when $\rho_{c}$ is increased, the concentrations of uninfected cells, actively infected 


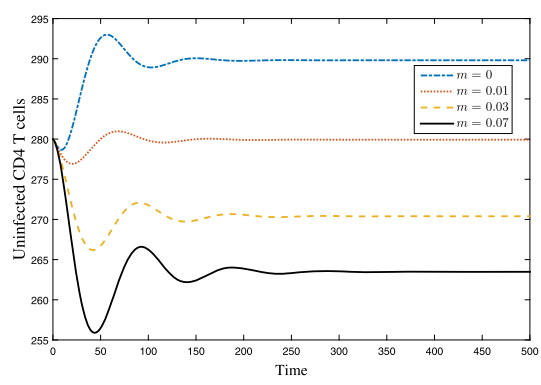

(a) The concentration of uninfected CD4 T cells

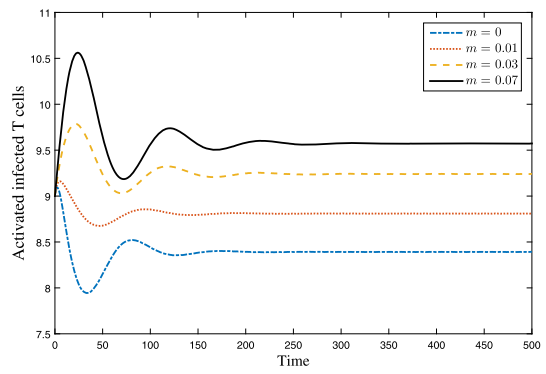

(c) The concentration of actively infected CD4 T cells

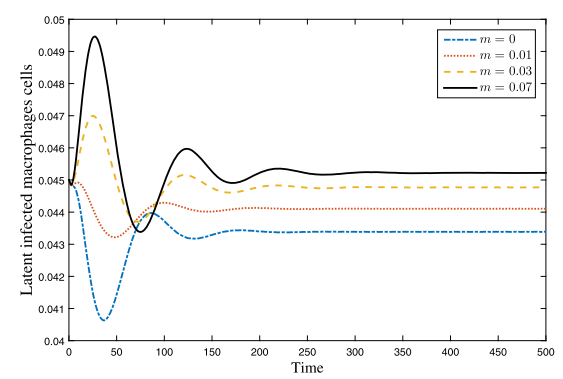

(e) The conc. of latently infected macrophages cells

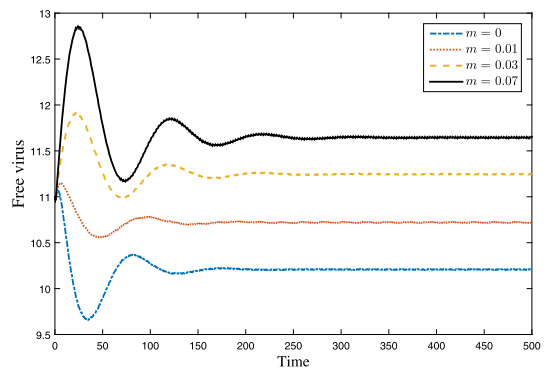

(g) The concentration of free virus

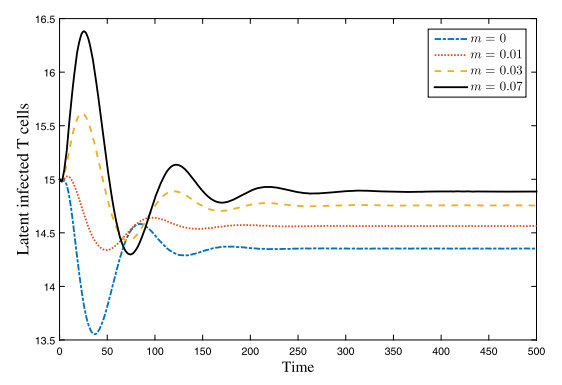

(b) The concentration of latently infected CD4 T cells

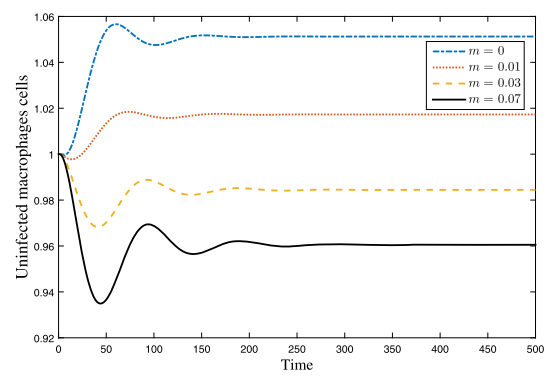

(d) The conc. of uninfected macrophages cells

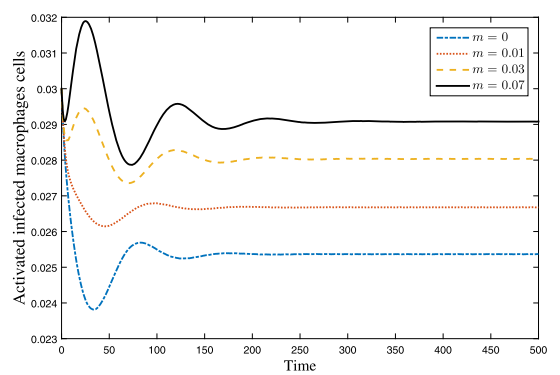

(f) The conc. of actively infected macrophages cells

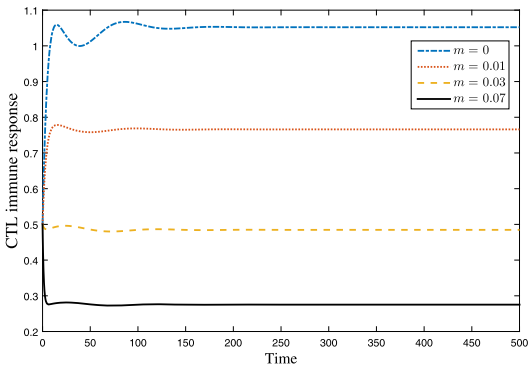

(h) The concentration of CTL immune response

Figure 3 The evolution of model (6)-(13) with varied $m$

cells, free HIV particles and CTLs are decreased. Using the values of the parameters given in Table 1 we have the following:

(i) if $0.0 \leq \rho_{c}<0.9437$, then $R_{0}>1$ and $E^{*}$ exists and is globally asymptomatically stable;

(ii) if $\rho_{c} \geq 0.9437$, then $R_{0} \leq 1$ and $E_{0}$ is globally asymptomatically stable.

Case IV. Effect of the immune impairment parameter $m$ on the HIV dynamics 


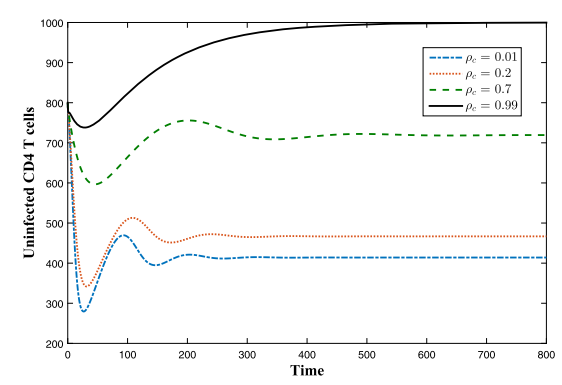

(a) The concentration of uninfected CD4 T cells

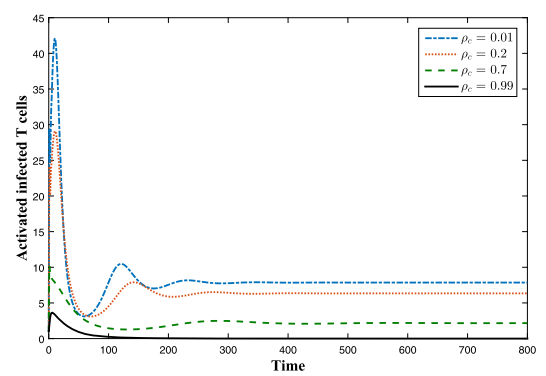

(c) The concentration of actively infected CD4 T cells

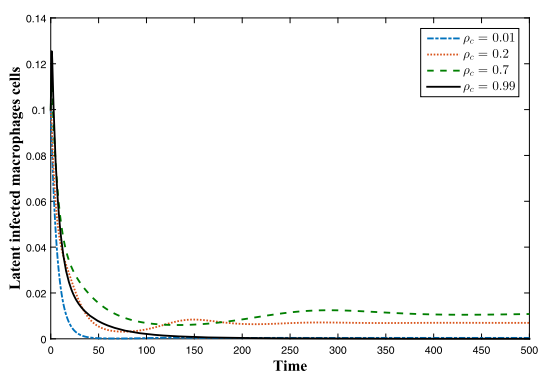

(e) The conc. of latently infected macrophages cells

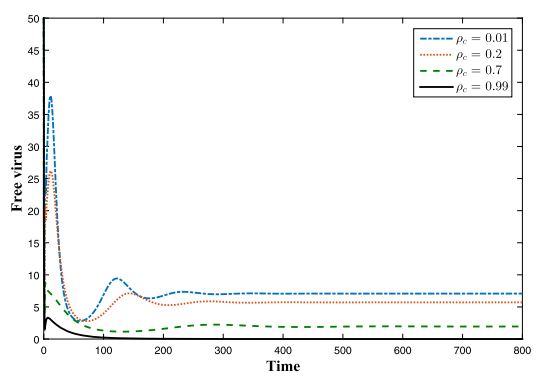

(g) The concentration of free virus

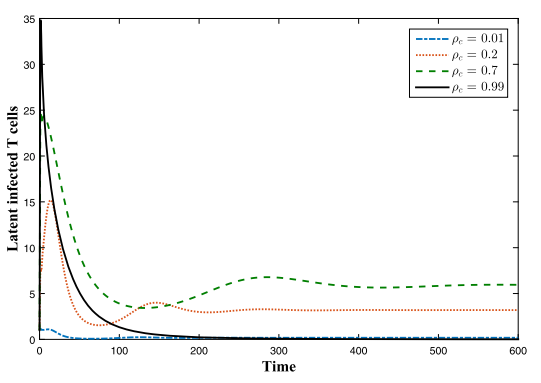

(b) The concentration of latently infected CD4 T cells

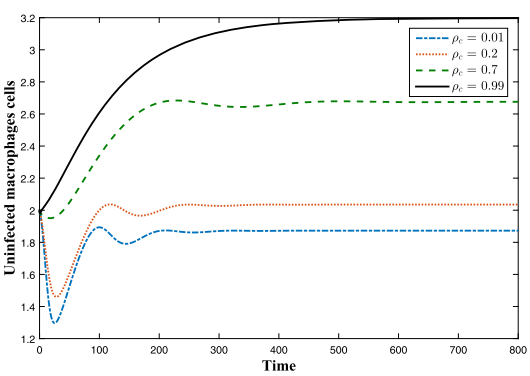

(d) The conc. of uninfected macrophages cells

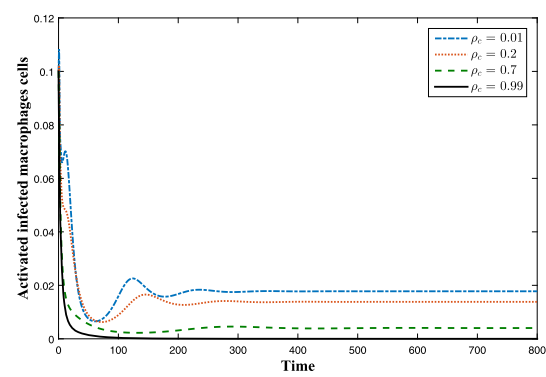

(f) The conc. of actively infected macrophages cells

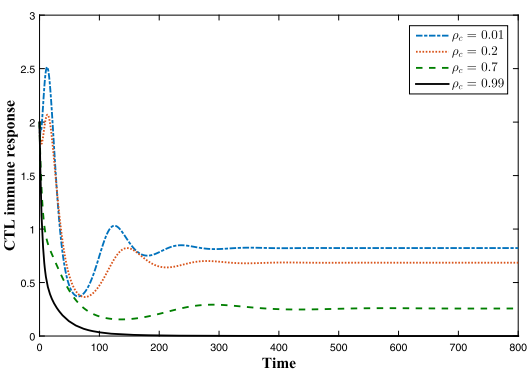

(h) The concentration of CTL immune response

Figure 4 The evolution of model (6)-(13) with varied $\rho_{c}$

In this case, we choose $\beta_{1}=2.4 \times 10^{-3}, \beta_{2}=2 \times 10^{-3}, \tau_{c}=0.5, \rho_{c}=0.5$ and the initial conditions IC4: $\omega_{1}(\eta)=280, \omega_{2}(\eta)=15, \omega_{3}(\eta)=9, \omega_{4}(\eta)=1, \omega_{5}(\eta)=0.045, \omega_{6}(\eta)=$ $0.03, \omega_{7}(\eta)=11$ and $\omega_{8}(\eta)=0.5$. Figure 3 shows that, as $m$ is increased, the concentration of CTL cells is decreased and then the concentrations of latently infected cells, actively infected cells and free HIV particles are increased, while the concentration of the unin- 
fected cells is decreased. We note that $R_{0}$ does not depend on the parameter $m$; therefore, $m$ does not change the stability properties of steady states.

\section{Conclusion and discussion}

All of the existing mathematical models of HIV infection with CTL immune impairment study the HIV infection and production in one class of target cells, CD4 ${ }^{+} \mathrm{T}$ cells. However, it has been reported in several papers that HIV can infect both $\mathrm{CD} 4^{+} \mathrm{T}$ cells and macrophages. In this paper, we have studied an HIV dynamics model with CTL immune impairment and with two classes of target cells, $\mathrm{CD} 4^{+} \mathrm{T}$ cells and macrophages. We have considered two types of infected cells, latently infected cells (such cells contain HIV but are not producing it) and actively infected cells (such cells are producing HIV). The model considers multiple discrete or distributed time delays to characterize the time between an HIV contact of an uninfected target cell and the creation of mature HIV particles. We have shown that the solutions of the model are nonnegative and ultimately bounded which ensures the well-posedness of the model. We have derived a biological threshold number $R_{0}$ (the basic reproduction number) which fully determines the existence and stability of the two steady states of the model. We have investigated the global stability of the model steady states by using Lyapunov method and LaSalle's invariance principle. We have proven that (i) if $R_{0} \leq 1$, then the infection-free steady state $E_{0}$ is globally asymptotically stable and HIV is predicted to be completely cleared from the HIV infected individuals, (ii) if $R_{0}>1$, then the chronic steady state $E^{*}$ is globally asymptotically stable and a chronic HIV infection is attained. We have conducted numerical simulations and have shown that both the theoretical and numerical results are consistent.

Our analysis extends the results presented in [49], where the global stability was analyzed for a model with one target cell population. When we consider the HIV dynamics with only one class of target cells, CD4 ${ }^{+}$T cells, then model (6)-(13) under the effect of RTI drug therapy with drug efficacy $\epsilon$ leads to the following model:

$$
\begin{aligned}
& \dot{x}_{1}(t)=\lambda_{1}-d_{1} x_{1}(t)-(1-\epsilon) \beta_{1} x_{1}(t) v(t), \\
& \dot{y}_{1}(t)=(1-\epsilon) \rho_{1} \beta_{1} e^{-\alpha_{1} \tau_{1}} x_{1}\left(t-\tau_{1}\right) v\left(t-\tau_{1}\right)-a y_{1}(t)-\delta y_{1}(t), \\
& \dot{u}_{1}(t)=(1-\epsilon)\left(1-\rho_{1}\right) \beta_{1} e^{-\alpha_{2} \tau_{2}} x_{1}\left(t-\tau_{2}\right) v\left(t-\tau_{2}\right)+\delta y_{1}(t)-p u_{1}(t) z(t)-\mu u_{1}(t), \\
& \dot{v}(t)=k e^{-\alpha_{5} \tau_{5}} u_{1}\left(t-\tau_{5}\right)-r v(t), \\
& \dot{z}(t)=c u_{1}(t)-m u_{1}(t) z(t)-b z(t) .
\end{aligned}
$$

The basic reproduction number for system (44)-(48) is given by

$$
R_{0}^{C}(\epsilon)=\frac{(1-\epsilon) \delta k \gamma_{1} \beta_{1} x_{1}^{0}}{\mu r(a+\delta)}
$$

The basic reproduction number for system (6)-(13) under the effect of RTI drug therapy can be written as:

$$
\begin{aligned}
& R_{0}(\epsilon)=R_{0}^{C}(\epsilon)+R_{0}^{M}(\epsilon), \\
& R_{0}^{C}(\epsilon)=\frac{(1-\epsilon) \delta k \gamma_{1} \beta_{1} x_{1}^{0}}{\mu r(a+\delta)},
\end{aligned}
$$




$$
R_{0}^{M}(\epsilon)=\frac{(1-\epsilon) \delta k \gamma_{2} \beta_{2} x_{2}^{0}}{\mu r(a+\delta)}
$$

where $R_{0}^{M}(\epsilon)$ is the basic reproduction number of a model that describes the HIV dynamics with only macrophages and neglects the $C D 4^{+} \mathrm{T}$ cells. For system (44)-(48) one can determine the minimum drug efficacy $\epsilon_{m}^{C}$ such that $R_{0}^{C}\left(\epsilon_{m}^{C}\right)<1$, namely

$$
\epsilon_{m}^{C}=\max \left\{1-\frac{\mu r(a+\delta)}{\delta k \gamma_{1} \beta_{1} x_{1}^{0}}, 0\right\}
$$

Comparing Eqs. (43) and (49), we get that $\epsilon_{m}^{C} \leq \epsilon_{m}$. Therefore, if we apply drugs with $\epsilon$ such that $\epsilon_{m}^{C} \leq \epsilon<\epsilon_{m}$, this guarantees that $R_{0}^{C}(\epsilon) \leq 1$, and then $E_{0}$ of system (44)-(48) is globally asymptotically stable; however, $R_{0}(\epsilon)>1$ and so $E_{0}$ of (6)-(13) is unstable. Therefore, more accurate drug efficacy $\epsilon$ is determined when using the model with two classes of target cells. This shows the importance of considering the effect of the macrophages in the HIV dynamics.

In the literature, fractional-order differential equations have been applied with the purpose of obtaining a deeper understanding of the complex behavioral patterns of HIV dynamical systems [53, 55-57]. The memory property of the fractional models allows the integration of more information from the past, which translates into more accurate predictions for the model. Clinicians can thus use the information (in terms of behavior predictions) of fractional-order systems to fit patients' data with the most appropriate noninteger-order index [53]. As a future work it is reasonable to use a corresponding fractional modification of our models, as fractional differential equations inherently include memory.

Funding

This article was funded by King Abdulaziz City for Science and Technology (KACST), Saudi Arabia, under grant No. (1-17-01-009-0103). The authors, therefore, acknowledge with thanks KACST for technical and financial support.

Availability of data and materials

Not applicable.

Competing interests

The authors declare that they have no competing interests.

Authors' contributions

All authors contributed equally to the writing of this paper. All authors read and approved the final manuscript.

Author details

${ }^{1}$ Department of Mathematics, Faculty of Science, King Abdulaziz University, Jeddah, Saudi Arabia. ${ }^{2}$ Department of Mathematics, Faculty of Science, King Khalid University, Abha, Saudi Arabia. ${ }^{3}$ Department of Mathematics, Faculty of Science, Assiut University, Assiut, Egypt.

\section{Publisher's Note}

Springer Nature remains neutral with regard to jurisdictional claims in published maps and institutional affiliations.

Received: 6 July 2018 Accepted: 31 October 2018 Published online: 09 November 2018

\section{References}

1. Nowak, M.A., Bangham, C.R.M.: Population dynamics of immune responses to persistent viruses. Science 272, 74-79 (1996)

2. Culshaw, R.V., Ruan, S.: A delay-differential equation model of HIV infection of CD4+T-cells. Math. Biosci. 165, 27-39 (2000)

3. Nelson, P.W., Murray, J.D., Perelson, A.S.: A model of HIV-1 pathogenesis that includes an intracellular delay. Math. Biosci. 163, 201-215 (2000)

4. Culshaw, R.V., Ruan, S., Webb, G.: A mathematical model of cell-to-cell spread of HIV-1 that includes a time delay. J. Math. Biol. 46, 425-444 (2003) 
5. Wang, L., Li, M.Y.: Mathematical analysis of the global dynamics of a model for HIV infection of CD4 ${ }^{+} \mathrm{T}$ cells. Math. Biosci. 200, 44-57 (2006)

6. Zhao, Y., Dimitrov, D.T., Liu, H., Kuang, Y.: Mathematical insights in evaluating state dependent effectiveness of HIV prevention interventions. Bull. Math. Biol. 75(4), 649-675 (2013)

7. Nelson, P.W., Murray, J.D., Perelson, A.S.: A model of HIV-1 pathogenesis that includes an intracellular delay. Math. Biosci. 163(2), 201-215 (2000)

8. Huang, G., Takeuchi, Y., Ma, W.: Lyapunov functionals for delay differential equations model of viral infections. SIAM J. Appl. Math. 70(7), 2693-2708 (2010)

9. Elaiw, A.M., AlShamrani, N.H.: Global properties of nonlinear humoral immunity viral infection models. Int. J. Biomath. 8(5), Article ID 1550058 (2015)

10. Elaiw, A.M., AlShamrani, N.H., Hattaf, K.: Dynamical behaviors of a general humoral immunity viral infection model with distributed invasion and production. Int. J. Biomath. 10(3), Article ID 1750035 (2017)

11. Elaiw, A.M., AlShamrani, N.H.: Stability of a general delay-distributed virus dynamics model with multi-staged infected progression and immune response. Math. Methods Appl. Sci. 40(3), 699-719 (2017)

12. Elaiw, A.M., Elnahary, E.Kh., Raezah, A.A.: Effect of cellular reservoirs and delays on the global dynamics of HIV. Adv. Differ. Equ. 2018, 85 (2018)

13. Hattaf, K., Yousfi, N.: A generalized virus dynamics model with cell-to-cell transmission and cure rate. Adv. Differ. Equ. 2016, 174 (2016)

14. Elaiw, A.M., Raezah, A.A., Hattaf, K.: Stability of HIV-1 infection with saturated virus-target and infected-target incidences and CTL immune response. Int. J. Biomath. 10(5), Article ID 1750070 (2017)

15. Elaiw, A.M., AIShamrani, N.H., Alofi, A.S.: Stability of CTL immunity pathogen dynamics model with capsids and distributed delay. AIP Adv. 7, Article ID 125111 (2017)

16. Gibelli, L., Elaiw, A., Alghamdi, M.A., Althiabi, A.M.: Heterogeneous population dynamics of active particles: progression, mutations, and selection dynamics. Math. Models Methods Appl. Sci. 27, 617-640 (2017)

17. Wang, J., Teng, Z., Miao, H.: Global dynamics for discrete-time analog of viral infection model with nonlinear incidence and CTL immune response. Adv. Differ. Equ. 2016, 143 (2016)

18. Blankson, J.N., Persaud, D., Siliciano, R.F.: The challenge of viral reservoirs in HIV-1 infection. Annu. Rev. Med. 53, 557-593 (2002)

19. Elaiw, A.M., Raezah, A.A.: Stability of general virus dynamics models with both cellular and viral infections and delays. Math. Methods Appl. Sci. 40(16), 5863-5880 (2017)

20. Elaiw, A.M., Raezah, A., Alofi, A.S.: Effect of humoral immunity on HIV-1 dynamics with virus-to-target and infected-to-target infections. AIP Adv. 6(8), Article ID 085204 (2016)

21. Elaiw, A.M., Raezah, A., Alofi, A.: Stability of a general delayed virus dynamics model with humoral immunity and cellular infection. AIP Adv. 7(6), Article ID 065210 (2017)

22. Dutta, A., Gupta, P.K.: A mathematical model for transmission dynamics of HIV/AIDS with effect of weak CD4+ $T$ cells. Chin. J. Phys. (Accepted)

23. Buonomo, B., Vargas-De-León, C.: Global stability for an HIV-1 infection model including an eclipse stage of infected cells. J. Math. Anal. Appl. 385, 709-720 (2012)

24. Perelson, A.S., Kirschner, D.E., Boer, R.D.: Dynamics of HIV infection of CD4+ T cells. Math. Biosci. 114(1), 81-125 (1993)

25. Korobeinikov, A.: Global properties of basic virus dynamics models. Bull. Math. Biol. 66(4), 879-883 (2004)

26. Elaiw, A.M., AlShamrani, N.H.: Global stability of humoral immunity virus dynamics models with nonlinear infection rate and removal. Nonlinear Anal., Real World Appl. 26, 161-190 (2015)

27. Monica, C., Pitchaimani, M.: Analysis of stability and Hopf bifurcation for HIV-1 dynamics with PI and three intracellular delays. Nonlinear Anal., Real World Appl. 27, 55-69 (2016)

28. Li, M.Y., Wang, L.: Backward bifurcation in a mathematical model for HIV infection in vivo with anti-retroviral treatment. Nonlinear Anal., Real World Appl. 17, 147-160 (2014)

29. Li, B., Chen, Y., Lu, X., Liu, S.: A delayed HIV-1 model with virus waning term. Math. Biosci. Eng. 13, 135-157 (2016)

30. Perelson, A.S., Nelson, P.W.: Mathematical analysis of HIV-1 dynamics in vivo. SIAM Rev. 41(1), 3-44 (1999)

31. Perelson, A.S., Essunger, P., Cao, Y., Vesanen, M., Hurley, A., Saksela, K., Markowitz, M., Ho, D.D.: Decay characteristics of HIV-1-infected compartments during combination therapy. Nature 387, 188-191 (1997)

32. Callaway, D.S., Perelson, A.S.: HIV-1 infection and low steady state viral loads. Bull. Math. Biol. 64, 29-64 (2002)

33. Adams, B.M., Banks, H.T., Davidian, M., Kwon, H.-D., Tran, H.T., Wynne, S.N., Rosenberg, E.S.: HIV dynamics: modeling, data analysis, and optimal treatment protocols. J. Comput. Appl. Math. 184, 10-49 (2005)

34. Elaiw, A.M.: Global properties of a class of HIV models. Nonlinear Anal., Real World Appl. 11, 2253-2263 (2010)

35. Elaiw, A.M.: Global properties of a class of virus infection models with multitarget cells. Nonlinear Dyn. 69(1-2), 423-435 (2012)

36. Elaiw, A.M., Almuallem, N.A.: Global dynamics of delay-distributed HIV infection models with differential drug efficacy in cocirculating target cells. Math. Methods Appl. Sci. 39, 4-31 (2016)

37. Elaiw, A.M., Hassanien, I.A., Azoz, S.A.: Global stability of HIV infection models with intracellular delays. J. Korean Math. Soc. 49(4), 779-794 (2012)

38. Elaiw, A.M., Azoz, S.A.: Global properties of a class of HIV infection models with Beddington-DeAngelis functional response. Math. Methods Appl. Sci. 36, 383-394 (2013)

39. Elaiw, A.M., Almuallem, N.A.: Global properties of delayed-HIV dynamics models with differential drug efficacy in cocirculating target cells. Appl. Math. Comput. 265, 1067-1089 (2015)

40. Pinto, C.M.A., Carvalho, A.R.M.: A latency fractional order model for HIV dynamics. J. Comput. Appl. Math. 312, 240-256 (2017)

41. Shu, H., Wang, L., Watmough, J.: Global stability of a nonlinear viral infection model with infinitely distributed intracellular delays and CTL immune responses. SIAM J. Appl. Math. 73(3), 1280-1302 (2013)

42. Wang, X., Elaiw, A.M., Song, X.: Global properties of a delayed HIV infection model with CTL immune response. Appl. Math. Comput. 218(18), 9405-9414 (2012)

43. Huang, D., Zhang, X., Guo, Y., Wang, H.: Analysis of an HIV infection model with treatments and delayed immune response. Appl. Math. Model. 40(4), 3081-3089 (2016) 
44. Ali, N., Zaman, G., Algahtani, O.: Stability analysis of HIV-1 model with multiple delays. Adv. Differ. Equ. 2016, 88 (2016). https://doi.org/10.1186/s13662-016-0808-4

45. Iwami, S., Nakaoka, S., Takeuchi, Y., Miura, Y., Miura, T.: Immune impairment thresholds in HIV infection. Immunol. Lett. 123(2), 149-154 (2009)

46. Hu, Z., Zhang, J., Wang, H., Ma, W., Liao, F.: Dynamics analysis of a delayed viral infection model with logistic growth and immune impairment. Appl. Math. Model. 38, 524-534 (2014)

47. Regoes, R., Wodarz, D., Nowak, M.A.: Virus dynamics: the effect to target cell limitation and immune responses on virus evolution. J. Theor. Biol. 191, 451-462 (1998)

48. Krishnapriya, P., Pitchaimani, M.: Modeling and bifurcation analysis of a viral infection with time delay and immune impairment. Jpn. J. Ind. Appl. Math. 34, 99-139 (2017)

49. Elaiw, A.M., Raezah, A.A., Alofi, B.S.: Dynamics of delayed pathogen infection models with pathogenic and cellular infections and immune impairment. AIP Adv. 8, Article ID 025323 (2018)

50. Avila-Vales, E., Chan-Chí, N., García-Almeida, G.: Analysis of a viral infection model with immune impairment, intracellular delay and general non-linear incidence rate. Chaos Solitons Fractals 69, 1-9 (2014)

51. Wang, S., Song, X., Ge, Z:: Dynamics analysis of a delayed viral infection model with immune impairment. Appl. Math. Model. 35(10), 4877-4885 (2011)

52. Krishnapriya, P., Pitchaimani, M.: Analysis of time delay in viral infection model with immune impairment. J. Appl. Math. Comput. 55, 421-453 (2017)

53. Carvalho, A.R.M., Pinto, C.M.A., Baleanu, D.: HIV/HCV coinfection model: a fractional-order perspective for the effect of the HIV viral load. Adv. Differ. Equ. 2018, 2 (2018)

54. Hale, J.K., Lunel, S.M.V.: Introduction to Functional Differential Equations. Springer, New York (1993)

55. Carvalho, A.R.M., Pinto, C.M.A.: Contributions of the latent reservoir and of the pool of long-lived chronically infected CD4+ T cells in HIV dynamics: a fractional approach. Proceedings of the ENOC2017, June 25-30, 2017, Budapest, Hungary

56. Yan, Y., Kou, C.: Stability analysis for a fractional differential model of HIV infection of $\mathrm{CD} 4^{+} \mathrm{T}$-cells with time delay. Math. Comput. Simul. 82, 1572-1585 (2012)

57. Jajarmi, A., Baleanu, D.: A new fractional analysis on the interaction of HIV with CD4+ T-cells. Chaos Solitons Fractals $113,221-229(2018)$

58. LV, C., Huang, L., Yuan, Z:: Global stability for an HIV-1 infection model with Beddington-DeAngelis incidence rate and CTL immune response. Commun. Nonlinear Sci. Numer. Simul. 19, 121-127 (2014)

59. Shi, X., Zhou, X., Son, X.: Dynamical behavior of a delay virus dynamics model with CTL immune response. Nonlinear Anal., Real World Appl. 11, 1795-1809 (2010)

\section{Submit your manuscript to a SpringerOpen ${ }^{\circ}$ journal and benefit from:}

- Convenient online submission

- Rigorous peer review

- Open access: articles freely available online

- High visibility within the field

- Retaining the copyright to your article

Submit your next manuscript at $\boldsymbol{~ s p r i n g e r o p e n . c o m ~}$ 